\title{
Targeted Synovial Fluid Proteomics for Biomarker Discovery in Rheumatoid Arthritis
}

\author{
Leticia Cano • Daniel G. Arkfeld
}

Published online: 23 May 2009

(C) Humana Press 2009

\begin{abstract}
Objective Rheumatoid arthritis (RA) is an autoimmune disease that targets the synovium. The autoantigens involved in the autoantibody responses in RA are unknown. A targeted proteomics approach was used to identify proteins in RA synovial fluid (SF) that are recognized by autoantibodies in RA sera.

Methods RA SF, depleted of abundant proteins, was fractionated by two-dimensional liquid chromatography (chromatofocusing followed by reverse phase HPLC). Protein arrays constructed from these fractions were probed with RA and normal control sera, and proteins within reactive fractions were identified by mass spectrometry. The reactivity of RA sera to an identified peptide was confirmed by ELISA.
\end{abstract}

Electronic supplementary material The online version of this article (doi:10.1007/s12014-009-9028-1) contains supplementary material, which is available to authorized users.

L. Cano $(\bowtie)$

Division of Immunology, Beckman Research Institute of the

City of Hope National Medical Center,

Duarte, CA 91010, USA

e-mail: canol@nhlbi.nih.gov

D. G. Arkfeld

Division of Rheumatology, Department of Medicine,

University of Southern California Keck School of Medicine and

the Los Angeles County and University of Southern California

Medical Center,

Los Angeles, CA 90033, USA

Present address:

L. Cano

Laboratory of Applied Mass Spectrometry, National Heart,

Lung and Blood Institute, National Institutes of Health,

Bethesda, MD, USA
Results RA sera specifically reacted to a SF fraction containing fibrin. Mass spectrometry analyses established the presence of a citrullinated arginine at position 271 of the fibrin fragment present in RA SF. A synthetic peptide corresponding to fibrin residues 259-287, containing the citrulline substitution at Arg 271, was recognized by 10 of 12 RA sera, but by two of 18 normal control sera and one of 10 systemic lupus erythematosus sera.

Conclusion Proteomics methodology can be used to directly characterize post-translational modifications in candidate autoantigens isolated from sites of disease activity. The finding that RA sera contain antibodies to the citrullinated fibrin 259-287 peptide may ultimately lead to improved diagnostic tests for RA and/or biomarkers for disease activity.

Keywords Rheumatoid arthritis · Synovial fluid ·

Clinical proteomics - Biomarker discovery - Citrullination .

Fibrinogen · ELISA, protein macroarrays .

Autoimmune disease - Autoantibodies - Autoantigens .

Mass spectrometry $\cdot$ Post-translational modification

\section{Introduction}

Rheumatoid arthritis (RA) is an autoimmune disease that targets the joints and affects $0.8 \%$ of the adults worldwide $[1,2]$. Chronic joint inflammation leads to cartilage and bone destruction, resulting in loss of function. Many selfantigens have been implicated in the triggering and/or maintenance of autoreactive lymphocyte responses in RA [3-5]. Nevertheless, there remains an uncertainty as to how disease is caused and maintained [6]. Aberrant posttranslational modifications of self-proteins may play a role in breaking $\mathrm{T}$ and $\mathrm{B}$ cell tolerance, leading to autoimmunity [7-9]. Of particular interest to the clinical management of 
RA are the anti-citrulline antibodies [10], which can predict both development $[11,12]$ and severity of disease $[13,14]$. Citrullination is the post-translational modification (deimination) of arginine to citrulline catalyzed by protein arginyl deiminase (PAD) enzymes [15]. This conversion changes the charge of the site from a positive to a neutral and increases the mass of the amino acid by $1 \mathrm{Da}$. The difference in charge may cause protein unfolding [16], thereby exposing novel epitopes.

The current diagnostic test for anti-citrulline antibodies employs a cyclic citrullinated peptide (CCP), yet the citrulline residues on synovial joint proteins that are target(s) of anti-citrulline antibodies in vivo have not been precisely defined. Autoantigens, which exist in citrullinated forms include fibrinogen [17-19] (which was initially thought to be filaggrin $[20,21]$ ), vimentin [22-24], collagen type I [25, 26], collagen type II [2628], fibronectin [29], and alpha-enolase [30]. The presence of citrulline-modified fibrinogen alpha (FIBA) and beta chains in RA synovial tissue or fluid has been reported [17, 19].

The goal of this project was to develop a method to discover novel RA autoantigens using a targeted proteomic analysis. We and others have reasoned that autoantigens might be enriched in RA SF and specifically recognized by autoantibodies in RA sera. Previous autoantigen and biomarker discovery projects have employed one of several approaches to fractionate biological sample preparations, including two-dimensional PAGE, miniaturized chips with diverse surfaces to promote differential protein binding, and multidimensional LC-MS/MS [31]. Often these fractionation approaches were combined with immunoblotting with patient sera. Although they yielded some novel information, such methods were complicated by the wide dynamic range of protein concentrations in serum and SF, which obscures identification of potentially informative proteins in minor abundance.

To separate and probe SF proteins, we used a method that was previously used to characterize cancer antigens [32], which included two-dimensional liquid chromatography, protein arrays, and high-resolution mass spectrometry. Depletion of abundant serum proteins followed by protein fractionation via two-dimensional liquid chromatography increased the likelihood of identifying the lower abundance proteins. Protein arrays constructed from fractionated SF were probed with RA and control sera to identify biologically significant fractions, and further analyses were performed only on those targeted fractions. It is important to note that the arrays were constructed from clinical samples that contain proteins with their post-translational modifications acquired in vivo. Liquid chromatography-tandem mass spectrometry (LC-MS/MS) was then used to identify immunogenic protein fragments and their post-translational modifications. Through this targeted proteomics approach, we have identified a citrulline-modified Arg 271 residue, within a fibrin alphaC domain fragment stably present in RA $\mathrm{SF}$, as a target of autoantibodies in RA sera.

\section{Materials and Methods}

\section{Patient Samples}

All samples were obtained using IRB approved protocols and all patients consented to be part of the study. SF and sera were obtained from patients receiving care in the outpatient Rheumatology clinics at the Los Angeles County and University of Southern California Medical Center. RA and systemic lupus erythematosus (SLE) patients were diagnosed according to established clinical criteria [33, 34]. All patients had to have a clinical diagnosis of RA as defined by rheumatologists at academic center. The sampling was on consecutive RA patients from LA County hospital who had a joint effusion that was aspirated. Due to use of biologic agents, much less effusions are seen in the clinic. Patient population was mostly Hispanic, approximately $80 \%$, with active RA stage 2 to 4 with most probably falling into 2 or 3 but data not collected so this is speculative. Comorbidities were not examined. All samples processed within $4 \mathrm{~h}$. Control sera were obtained from healthy volunteers at the City of Hope General Clinical Research Center. SF samples were diluted 1:5 in PBS, centrifuged to remove cellular debris, and stored at $-80^{\circ} \mathrm{C}$. There are no viscosity issues when the SF is diluted 1:5 in PBS. Blood samples were allowed to clot overnight at $4{ }^{\circ} \mathrm{C}$. The next day, the blood was centrifuged and the top layer of serum was transferred into new tubes. Serum samples were stored at $-80^{\circ} \mathrm{C}$ until use.

\section{Protein Fractionation}

A multiple affinity removal column (Agilent Technologies, Wilmington, DE, USA) was used to remove six abundant proteins (albumin, IgG, antitrypsin, IgA, transferrin, and haptoglobin) from SF. The procedure removed $85-90 \%$ of the total protein mass, which increased the probability of detecting the lower abundance proteins. Depletion was performed according to manufacturer's protocol. After the depletion, samples were desalted by use of a 5-kDa MWCO spin filter (Amicon Ultra-15, Millipore Corp., Bedford, MA, USA). Reactivity of RA sera to proteins in the $5-\mathrm{kDa}$ filtrate was not detected. Protein concentration of the desalted SF was determined by RCDC protein assay (BioRad Laboratories, Hercules, CA, USA).

SF proteins were separated by 2D-HPLC, chromatofocusing, and reverse phase (RP) HPLC. A Beckman PF2D

\section{菭 Humana Press}


System (Beckman Coulter, Inc., Fullerton, CA, USA) with a PF2D kit (column and buffers) was used for the firstdimension separation. Approximately $5 \mathrm{mg}$ of proteins was separated in the $\mathrm{pH}$ range 8.5 to 4.0. After loading, the sample was washed in start buffer for 20 min, eluant buffer for $75 \mathrm{~min}$, and then $1 \mathrm{M}$ sodium chloride buffer for $45 \mathrm{~min}$. The column was then washed overnight in water. Fractions were collected in increments of $5 \mathrm{~min}$ or $0.2 \mathrm{pH}$ units, whichever came first, into a cooled deep well 96-well plate. This separation was reproducible as performed according to manufacturers' supplied protocol. The second-dimension separation was performed on a Vydac C4 column ( 5 um, $300 \mathrm{~A}, 2.1 \times 250 \mathrm{~mm}$ ) using the following program: hold for $12 \mathrm{~min}$ at $5 \%$ buffer B; $5 \%$ to $95 \%$ buffer B in $25 \mathrm{~min}$; hold at $95 \%$ buffer B for $8 \mathrm{~min}$. The flow rate was $0.25 \mathrm{ml} / \mathrm{min}$. Fractions were collected between 14 and 45 min at every 2 min into standard 96well plates using a fraction collector.

The pooled SF second-dimension separations were performed on a Vydac C4 column (10 um, 300 A, 4.6× $250 \mathrm{~mm}$ ) using a gradient of $2 \%$ to $98 \%$ buffer B in $60 \mathrm{~min}$ and a flow rate of $1 \mathrm{ml} / \mathrm{min}$. Corresponding fractions that eluted in the same $\mathrm{pH}$ range from four first-dimension runs were combined to ensure enough material was obtained for analysis by mass spectrometry. Buffer A consisted of $0.1 \%$ TFA. Buffer B consisted of $0.1 \%$ TFA in $90 \%$ acetonitrile. A Beckman System Gold 126 equipped with a model 168 diode array detector (Beckman Coulter, Inc.) was used to perform the separations. Fractions were collected every minute. Samples were frozen and lyophilized to dryness.

\section{Protein Arrays}

Custom arrays were hand made using a VP 409 replicator with 96 pins each holding $100 \mathrm{nl}$ of fluid (V\&P Scientific, Inc., San Diego, CA, USA). The selected HPLC fractions were resuspended in $\sim 200 \mu \mathrm{l}$ of $6 \mathrm{M}$ urea/sodium bicarbonate $\mathrm{pH}$ 8.0. The microtiter plates were slowly rocked for about $30 \mathrm{~min}$ to facilitate protein solubility. The replicator was dipped into a 96-well plate, and the fluid $(\sim 100 \mathrm{nl})$ retained on the tips of the pins was transferred to a nitrocellulose membrane. Each fraction was arrayed in triplicate. To serve as a positive control for serum antibody reactivity, an influenza vaccine preparation also was arrayed. After drying overnight in a laminar flow hood, the arrayed membranes were blocked overnight in a non-fat dried milk solution and subsequently were rinsed twice in TBS (20 mM Tris-HCl, $500 \mathrm{mM} \mathrm{NaCl}, \mathrm{pH} 7.5)$. The arrays were incubated with a 1:200 dilution of RA or control sera for $1 \mathrm{~h}$ at room temperature. After rinsing twice in TBS, the arrays were incubated with a 1:100,000 dilution of HRPconjugated $\mathrm{F}\left(\mathrm{ab}^{\prime}\right) 2$ goat anti-human $\operatorname{IgG}+\operatorname{IgM}+\operatorname{IgA}$ secondary antibodies (Jackson ImmunoResearch Laborato- ries, Inc., West Grove, PA, USA). The arrays were washed twice with $3 \%$ newborn calf serum $/ 0.05 \%$ Tween/TBS, twice with a $0.05 \%$ Tween/TBS, twice with TBS, and twice with water. ECL Plus Western Blotting Detection Reagents (GE Healthcare, Piscataway, NJ, USA) were used as the detection agent. The arrays were imaged on a Typhoon 9410 (GE Healthcare) using the following conditions: Laser (457 nm), Emission filter (520BP40), Focal Plane (Platen), Pixel Size $(50 \mu \mathrm{m})$, Sensitivity (Normal). Different voltages were used to scan the images but typically $500 \mathrm{v}$ was used for most of the images. To distinguish background noise from foreground signal by a statistical method that complemented the visual inspection of the protein arrays, the median filter smoothing technique was applied to the imaged signals as described [35].

\section{Mass Spectrometry}

Approximately $80 \%$ of the solubilized RP-HPLC fraction was digested with trypsin (Promega, Madison, WI, USA). Approximately $5 \%$ of the digested material was analyzed by LC/MS/MS. Analyses were performed on a Thermo Finnigan LTQ-FT linear ion trap-Fourier transform mass spectrometer (Thermo Electron Corporation, San Jose, CA, USA) coupled to an Eksigent nanoLC-2D capillary HPLC system (Eksigent Technologies, LLC, Dublin, CA, USA). Samples were loaded onto a $300 \mu \mathrm{m} \times 5 \mathrm{~mm}$ C18 trapping column (Dionex Corporation, Sunnyvale, CA, USA) and then eluted through a lab-built $75 \mu \mathrm{m} \times 10 \mathrm{~cm}$ analytical column packed with $3 \mu \mathrm{m}$ C18 Pursuit resin (Varian, Inc., Palo Alto, CA, USA). The gradient for the trapping column was $100 \%$ A for $5 \mathrm{~min}$ using a flow rate of $10 \mu \mathrm{l} / \mathrm{min}$. The gradient for the analytical column was $2 \%$ to $35 \% \mathrm{~B}$ in $45 \mathrm{~min}, 35 \%$ to $50 \% \mathrm{~B}$ in $4 \mathrm{~min}$, and $50 \%$ to $95 \% \mathrm{~B}$ in 2 min using a flow rate of $0.2 \mu \mathrm{l} / \mathrm{min}$. High-resolution fullscale mass spectra were acquired in the Fourier transformion cyclotron resonance (FT-ICR) section of the mass spectrometer while fragment ion (MS/MS) spectra were obtained from the linear ion trap section. Fragmentation was performed using a collision energy setting of 35 . Dynamic exclusion was set at $15 \mathrm{~s}$.

Monoisotopic peaks and peptide charge states were determined during acquisition by the Xcalibur acquisition software using the high-resolution Fourier transform mass spectrometry (FTMS) spectra. Protein identifications were made by SEQUEST [36]. SEQUEST searches were performed with the following parameters: use of the 10/ 17/08 release of the SwissProt database (downloaded from ftp://ftp.ncbi.nlm.nih.gov/blast/db), monoisotopic masses, partial trypsin cleavage, 2 amu peptide and fragment tolerance, and automatic charge state determination. The SwissProt database was filtered to include only entries containing _ HUMAN as a parameter. Peptide hits were 


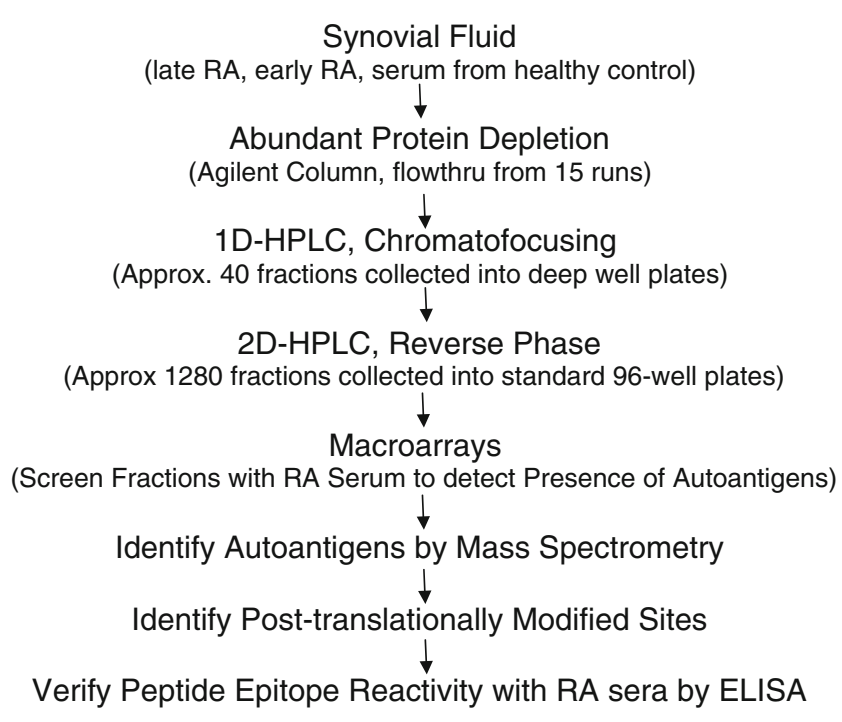

Fig. 1 Proteomic Strategy to Identify autoantigens present in synovial fluid of RA patients

filtered using the following criteria: DeltaCn greater than or equal to $0.08, \mathrm{XC}$ greater than or equal to 1.8 for peptides having +1 charge, 2.5 for peptides with a +2 charge, and 3.5 for peptides having a +3 charge and the peptide must be to a protein with probability score less than 0.0001 used [37]. Peptides meeting these criteria were further analyzed. Although the LTQ-FT has routine mass accuracy of $2 \mathrm{ppm}$, it is set to perform MS/MS on the most abundant peak in an isotope envelope, rather than the monoisotopic mass. This frequency results in errors of 1 or 2 mass units for large peptides. Because SEQUEST and extract_msn do not correct for this error, it was necessary to set peptide mass tolerance to 2.5 to account for errors in precursor assignment. Fragment ion tolerance was set at 0.0. The searches were performed assuming both trypsin and no enzyme specificity. Spectra were hand sorted to identify and verify post-translational modifications (PTM). All charge states and mass were manually verified using the high-resolution FTMS data. All spectra corresponding to candidate autoantigens were manually verified.

Synthetic Peptides

The FIBA 259-287 peptides with and without the citrulline substitution for arginine 271 (MELERPGGNEITR*GGSTSYGTGSETESPR) were synthesized in the City of Hope Peptide Synthesis Core Facility. The synthesized profilaggrin 619-631 peptide (SSFSQDR*DSQAQS) contained a citrulline for arginine substitution at position 625. Peptides were dissolved in $0.1 \%$ TFA and purified two to three times on a $\mathrm{C} 18$ reverse phase column. Concentration was determined by amino acid analysis.

\section{ELISA}

Maleic anhydride coated ELISA plates (Pierce Biotechnology, Inc., Rockford, IL, USA) were incubated overnight with $100 \mu \mathrm{l}$ of $12.5 \mu \mathrm{g} / \mathrm{ml}$ peptide in PBS. The plates were blocked overnight in a dried milk solution, washed twice in TBS, and incubated overnight in a 1:20 dilution of patient sera. After rinsing two times in TBS, the plates were incubated in a 1:10,000 dilution of HRP-conjugated $\mathrm{F}\left(\mathrm{ab}^{\prime}\right) 2$ goat anti-human IgG + IgM + IgA secondary antibodies for $1 \mathrm{~h}$. The plates were then rinsed 6 times in TBS. For color development $150 \mu \mathrm{l}$ ABTS (Pierce Biotechnology) was
Fig. 2 Fraction containing candidate autoantigen detected on second-dimension RP-HPLC. Fractions from the SF separation were spotted in a protein array, which was used to determine which fractions contained an autoantigen. Arrow points patient synovial fluid fraction that reacted with RA patient serum

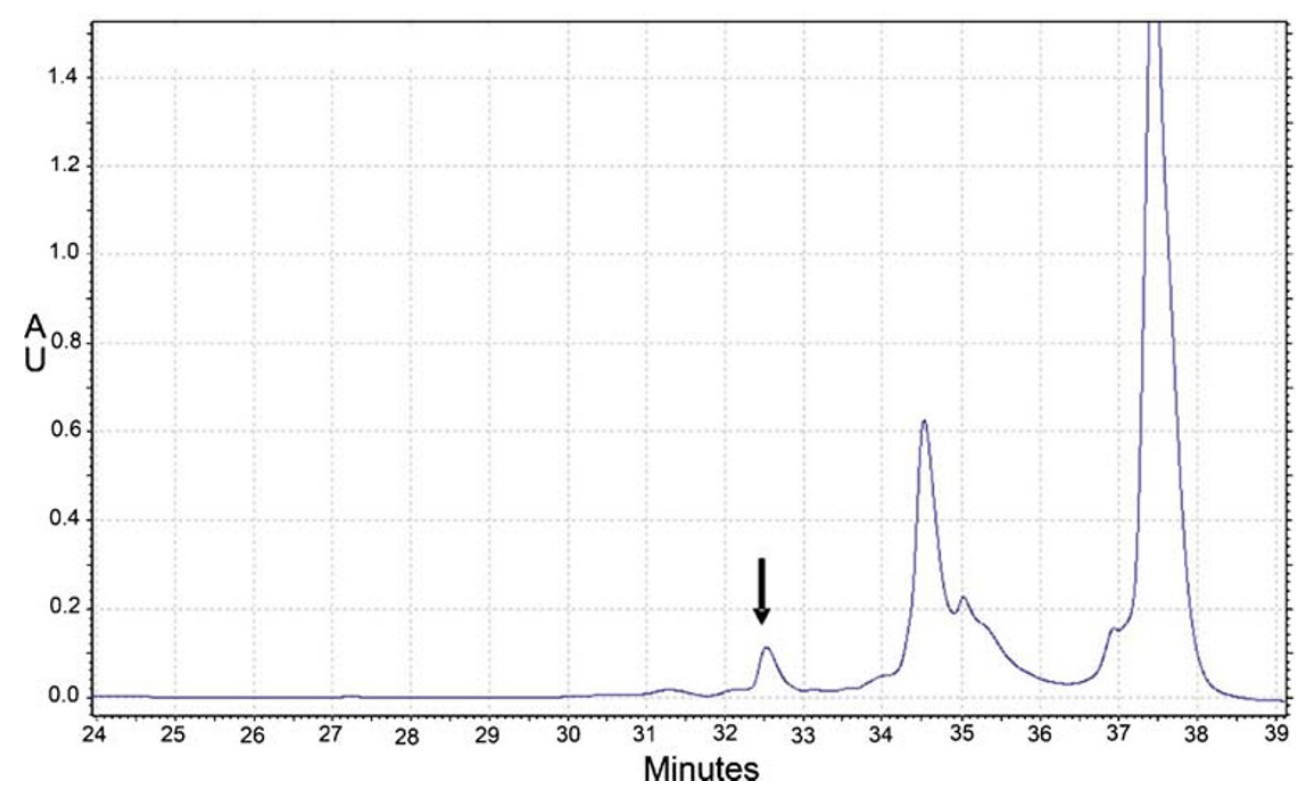


Fig. 3 a Representative HPLC chromatogram for the firstdimension chromatofocusing separation of the abundant protein depleted SF sample. The diagonal line indicates the observed $\mathrm{pH}$ gradient. The fractions corresponding to the elution range of $\mathrm{pH}$ 5.63-5.45 from four runs were combined for the second-dimension separation. b HPLC chromatogram for the second-dimension reverse phase separation. This sample was separated on a Beckman System Gold HPLC coupled to a diode array detector. The diagonal line indicates the solvent gradient $(\% \mathrm{~B})$. The fraction (between 20 and $25 \mathrm{~min}$ ) corresponding to the region that tested positive in the protein array assay was selected for further characterization by mass spectrometry a
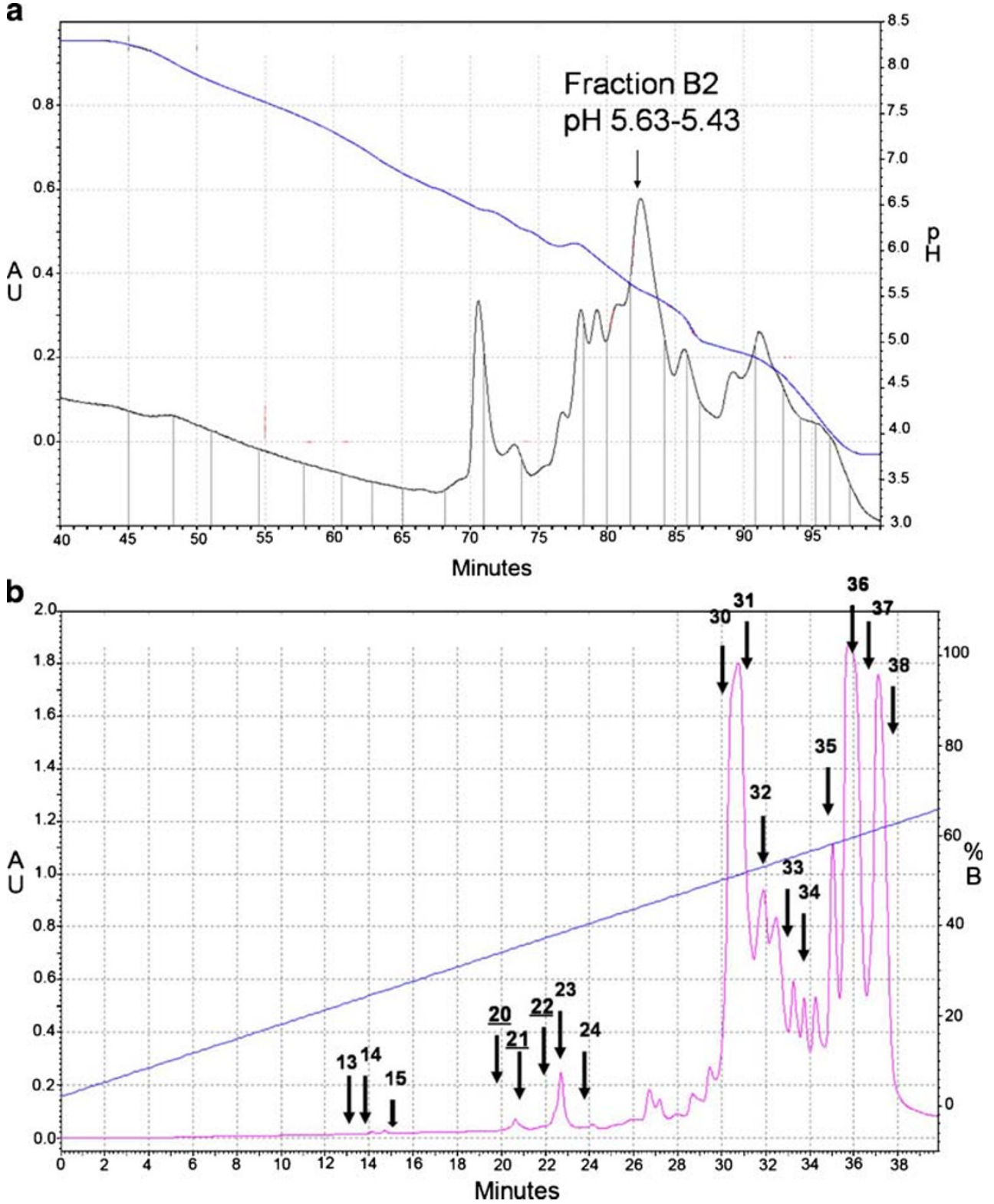

added to the wells and incubated for $30 \mathrm{~min}$. To stop the reaction, $100 \mu \mathrm{l}$ of $1 \%$ SDS was added. The absorbance at $405 \mathrm{~nm}$ was read on a plate reader.

\section{Results}

A targeted proteomic approach was performed to identify autoantigens in RA SF. The experimental design is diagrammed in Fig. 1. An immunodepletion column was first used to remove six abundant serum proteins (albumin, antitrypsin, haptoglobin, IgA, IgG, and transferrin). The depleted SF was then fractionated by chromatofocusing HPLC (provided as Supplement Fig. 1) and reverse phase
HPLC (provided as Supplement Fig. 2). This protein fractionation strategy increased the chance of identifying the lower abundance proteins by separating them from the higher abundance proteins. The second-dimension fractions were used to construct protein arrays on nitrocellulose membranes, which were used to test for the presence of autoantigens by analyzing differential reactivity of RA and control sera. We focused on one second-dimension fraction resulting from the separation of the first-dimension fraction eluting at $\mathrm{pH}$ 5.63-5.45 (Fig. 2) that tested positive when probed with RA serum but negative when probed with normal control serum. This was the only fraction that tested positive in which a peak was detected on the HPLC chromatographs. Although there was sufficient material for 
Table 1 Proteins identified in the B2 second-dimension RP fractions

\begin{tabular}{|c|c|c|c|c|}
\hline & $\mathrm{P}($ pro) & Score & MW & Hits \\
\hline \multicolumn{5}{|l|}{ Fraction 13 proteins } \\
\hline K1C9_HUMAN Keratin, type I cytoskeletal 9 & $3.93 \mathrm{E}-13$ & 60.40 & $62,091.8$ & 6 \\
\hline PRG4_HUMAN Proteoglycan-4 & $5.32 \mathrm{E}-13$ & 40.33 & $15,0,983.2$ & 5 \\
\hline GRP78_HUMAN 78 kDa glucose-regulated protein & $1.57 \mathrm{E}-09$ & 10.25 & $72,288.5$ & 1 \\
\hline ALBU_HUMAN Serum albumin & $2.20 \mathrm{E}-09$ & 40.24 & $69,321.6$ & 5 \\
\hline TYB4_HUMAN Thymosin beta-4 & $1.25 \mathrm{E}-07$ & 30.23 & $5,049.5$ & 6 \\
\hline K2C1_HUMAN Keratin, type II cytoskeletal 1 & $2.44 \mathrm{E}-07$ & 96.26 & $65,977.9$ & 10 \\
\hline K1C10_HUMAN Keratin, type I cytoskeletal 10 & $2.80 \mathrm{E}-07$ & 30.25 & $59,474.8$ & 3 \\
\hline \multicolumn{5}{|l|}{ Fraction 14 proteins } \\
\hline K1C9_HUMAN Keratin, type I cytoskeletal 9 & $1.00 \mathrm{E}-30$ & 110.27 & $62,091.8$ & 13 \\
\hline PRG4_HUMAN Proteoglycan-4 & $1.22 \mathrm{E}-14$ & 60.34 & $150,983.2$ & 11 \\
\hline K2C6C_HUMAN Keratin, type II cytoskeletal 6C & $1.33 \mathrm{E}-14$ & 10.31 & $59,988.4$ & 2 \\
\hline K2C1_HUMAN Keratin, type II cytoskeletal 1 & $2.93 \mathrm{E}-11$ & 126.39 & $65,977.9$ & 15 \\
\hline K1C10_HUMAN Keratin, type I cytoskeletal 10 & $7.90 \mathrm{E}-10$ & 10.26 & $59,474.8$ & 1 \\
\hline ALBU_HUMAN Serum albumin & $2.28 \mathrm{E}-09$ & 10.22 & $69,321.6$ & 2 \\
\hline TTHY_HUMAN Transthyretin & $3.41 \mathrm{E}-08$ & 20.26 & $15,877.1$ & 4 \\
\hline BASP_HUMAN Brain acid soluble protein 1 & $5.02 \mathrm{E}-07$ & 20.25 & $22,680.0$ & 2 \\
\hline EFHD2_HUMAN EF-hand domain-containing protein D2 & $1.42 \mathrm{E}-06$ & 10.19 & $26,680.5$ & 1 \\
\hline K22E_HUMAN Keratin, type II cytoskeletal 2 epidermal & $1.16 \mathrm{E}-05$ & 10.21 & $65,825.4$ & 1 \\
\hline APOA2_HUMAN Apolipoprotein A-II & $3.90 \mathrm{E}-04$ & 10.15 & $11,167.9$ & 1 \\
\hline \multicolumn{5}{|l|}{ Fraction 15 proteins } \\
\hline PRG4_HUMAN Proteoglycan-4 & $1.13 \mathrm{E}-11$ & 50.33 & $150,983.2$ & 8 \\
\hline K1C10_HUMAN Keratin, type I cytoskeletal 10 & $3.63 \mathrm{E}-10$ & 30.27 & $59,474.8$ & 3 \\
\hline K1C9_HUMAN Keratin, type I cytoskeletal 9 & $9.80 \mathrm{E}-08$ & 30.41 & $62,091.8$ & 3 \\
\hline FIBA_HUMAN Fibrinogen alpha chain & $7.51 \mathrm{E}-07$ & 30.22 & $94,914.3$ & 6 \\
\hline K2C1_HUMAN Keratin, type II cytoskeletal 1 & $6.51 \mathrm{E}-06$ & 70.26 & $65,977.9$ & 7 \\
\hline PMS2_HUMAN Mismatch repair endonuclease PMS2 & $2.41 \mathrm{E}-05$ & 10.19 & $95,737.1$ & 1 \\
\hline APOA1_HUMAN Apolipoprotein A-I & $3.14 \mathrm{E}-05$ & 30.13 & $30,758.9$ & 7 \\
\hline K22E_HUMAN Keratin, type II cytoskeletal 2 epidermal & $1.45 \mathrm{E}-04$ & 10.20 & $65,825.4$ & 1 \\
\hline S10A6_HUMAN Protein S100-A6 & $4.94 \mathrm{E}-04$ & 10.13 & $10,173.3$ & 1 \\
\hline \multicolumn{5}{|l|}{ Fraction 20 proteins } \\
\hline K2C6C_HUMAN Keratin, type II cytoskeletal 6C & $3.02 \mathrm{E}-12$ & 20.30 & $59,988.4$ & 3 \\
\hline FIBA_HUMAN Fibrinogen alpha chain & $4.08 \mathrm{E}-12$ & 408.35 & $94,914.3$ & 87 \\
\hline K2C1_HUMAN Keratin, type II cytoskeletal 1 & $2.09 \mathrm{E}-11$ & 106.30 & $65,977.9$ & 12 \\
\hline APOA4_HUMAN Apolipoprotein A-IV & $4.79 \mathrm{E}-11$ & 20.25 & $45,371.5$ & 3 \\
\hline K1C10_HUMAN Keratin, type I cytoskeletal 10 & $1.88 \mathrm{E}-10$ & 30.23 & $59,474.8$ & 3 \\
\hline K1C9_HUMAN Keratin, type I cytoskeletal 9 & $4.42 \mathrm{E}-10$ & 60.31 & $62,091.8$ & 6 \\
\hline ALBU_HUMAN Serum albumin & $5.87 \mathrm{E}-08$ & 20.21 & $69,321.6$ & 3 \\
\hline OSTP_HUMAN Osteopontin & $7.36 \mathrm{E}-07$ & 10.22 & $35,401.3$ & 1 \\
\hline APOA1_HUMAN Apolipoprotein A-I & $1.23 \mathrm{E}-05$ & 20.20 & $30,758.9$ & 2 \\
\hline K22E_HUMAN Keratin, type II cytoskeletal 2 epidermal & $6.83 \mathrm{E}-05$ & 10.22 & $65,825.4$ & 1 \\
\hline \multicolumn{5}{|l|}{ Fraction 21 proteins } \\
\hline K1C9_HUMAN Keratin, type I cytoskeletal 9 & $1.00 \mathrm{E}-30$ & 70.31 & $62,091.8$ & 8 \\
\hline K22E_HUMAN Keratin, type II cytoskeletal 2 epidermal & $5.55 \mathrm{E}-15$ & 30.28 & $65,825.4$ & 4 \\
\hline K2C6C_HUMAN Keratin, type II cytoskeletal 6C & $1.11 \mathrm{E}-14$ & 10.29 & $59,988.4$ & 2 \\
\hline K2C1_HUMAN Keratin, type II cytoskeletal 1 & $6.48 \mathrm{E}-13$ & 100.38 & $65,977.9$ & 12 \\
\hline FIBA_HUMAN Fibrinogen alpha chain & $8.06 \mathrm{E}-12$ & 428.33 & $94,914.3$ & 87 \\
\hline OSTP_HUMAN Osteopontin & $2.97 \mathrm{E}-09$ & 70.25 & $35,401.3$ & 14 \\
\hline K1C10_HUMAN Keratin, type I cytoskeletal 10 & $8.09 \mathrm{E}-09$ & 40.24 & $59,474.8$ & 4 \\
\hline
\end{tabular}

\section{藻 Humana Press}


Table 1 (continued)

\begin{tabular}{|c|c|c|c|c|}
\hline & $\mathrm{P}$ (pro) & Score & MW & Hits \\
\hline ADIPO_HUMAN Adiponectin & $2.01 \mathrm{E}-08$ & 10.22 & $26,397.0$ & 2 \\
\hline APOA1_HUMAN Apolipoprotein A-I & $3.54 \mathrm{E}-08$ & 110.20 & $30,758.9$ & 15 \\
\hline K2C5_HUMAN Keratin, type II cytoskeletal 5 & $4.79 \mathrm{E}-08$ & 10.25 & $62,340.0$ & 1 \\
\hline HEP2_HUMAN Heparin cofactor 2 & $1.86 \mathrm{E}-07$ & 20.26 & $57,034.3$ & 6 \\
\hline A2MG_HUMAN Alpha-2-macroglobulin & $4.97 \mathrm{E}-07$ & 10.22 & $163,174.3$ & 2 \\
\hline HPT_HUMAN Haptoglobin & $2.91 \mathrm{E}-06$ & 62.22 & $45,176.6$ & 11 \\
\hline APOB_HUMAN Apolipoprotein B-100 & $1.20 \mathrm{E}-05$ & 10.17 & $515,241.6$ & 2 \\
\hline APOA4_HUMAN Apolipoprotein A-IV & $1.46 \mathrm{E}-05$ & 20.19 & $45,371.5$ & 2 \\
\hline CLUS_HUMAN Clusterin & $5.35 \mathrm{E}-05$ & 10.16 & $52,461.1$ & 1 \\
\hline K2C75_HUMAN Keratin, type II cytoskeletal 75 & $1.47 \mathrm{E}-04$ & 16.20 & $59,468.0$ & 2 \\
\hline CD99_HUMAN CD99 antigen & $1.73 \mathrm{E}-04$ & 10.16 & $18,836.3$ & 1 \\
\hline \multicolumn{5}{|l|}{ Fraction 22 proteins } \\
\hline K22E_HUMAN Keratin, type II cytoskeletal 2 epidermal & $1.00 \mathrm{E}-30$ & 130.30 & $65,825.4$ & 17 \\
\hline K1C9_HUMAN Keratin, type I cytoskeletal 9 & $6.66 \mathrm{E}-15$ & 80.38 & $62,091.8$ & 9 \\
\hline K2C1_HUMAN Keratin, type II cytoskeletal 1 & $5.66 \mathrm{E}-14$ & 226.35 & $65,977.9$ & 34 \\
\hline HPT_HUMAN Haptoglobin & $1.12 \mathrm{E}-13$ & 98.26 & $45,176.6$ & 25 \\
\hline FIBA_HUMAN Fibrinogen alpha chain & $1.22 \mathrm{E}-11$ & 588.31 & $94,914.3$ & 115 \\
\hline K1C10_HUMAN Keratin, type I cytoskeletal 10 & $2.69 \mathrm{E}-11$ & 200.34 & $59,474.8$ & 34 \\
\hline K2C6C_HUMAN Keratin, type II cytoskeletal 6C & $4.30 \mathrm{E}-11$ & 50.31 & $59,988.4$ & 7 \\
\hline FETUA_HUMAN Alpha-2-HS-glycoprotein & $2.71 \mathrm{E}-09$ & 10.21 & $39,299.7$ & 1 \\
\hline PLMN_HUMAN Plasminogen & $3.35 \mathrm{E}-09$ & 20.28 & $90,510.2$ & 5 \\
\hline K2C5_HUMAN Keratin, type II cytoskeletal 5 & $7.18 \mathrm{E}-08$ & 20.22 & $62,340.0$ & 3 \\
\hline K1C14_HUMAN Keratin, type I cytoskeletal 14 & $9.30 \mathrm{E}-08$ & 20.17 & $51,589.5$ & 2 \\
\hline APOA1_HUMAN Apolipoprotein A-I & $1.80 \mathrm{E}-07$ & 158.21 & $30,758.9$ & 78 \\
\hline LBR_HUMAN Lamin-B receptor & $7.18 \mathrm{E}-07$ & 10.18 & $70,658.2$ & 1 \\
\hline UPAR_HUMAN Urokinase plasminogen activator surface receptor & $8.12 \mathrm{E}-07$ & 10.24 & $36,953.3$ & 1 \\
\hline FINC_HUMAN Fibronectin & $8.99 \mathrm{E}-07$ & 30.22 & $262,439.5$ & 4 \\
\hline ECM1_HUMAN Extracellular matrix protein 1 & $1.83 \mathrm{E}-06$ & 20.18 & $60,635.4$ & 2 \\
\hline K2C6A_HUMAN Keratin, type II cytoskeletal 6A & $1.85 \mathrm{E}-06$ & 10.13 & $60,008.3$ & 1 \\
\hline TMOD3_HUMAN Tropomodulin-3 & $2.02 \mathrm{E}-06$ & 10.20 & $39,570.3$ & 1 \\
\hline K1C24_HUMAN Keratin, type I cytoskeletal 24 & $3.28 \mathrm{E}-06$ & 10.16 & $55,053.5$ & 1 \\
\hline OSTP_HUMAN Osteopontin & $9.62 \mathrm{E}-06$ & 30.24 & $35,401.3$ & 6 \\
\hline K1C17_HUMAN Keratin, type I cytoskeletal 17 & $1.05 \mathrm{E}-05$ & 30.17 & $48,076.1$ & 3 \\
\hline APOB_HUMAN Apolipoprotein B-100 & $2.09 \mathrm{E}-05$ & 10.15 & $515,241.6$ & 1 \\
\hline K2C75_HUMAN Keratin, type II cytoskeletal 75 & $2.46 \mathrm{E}-05$ & 36.21 & $59,468.0$ & 4 \\
\hline CFAB_HUMAN Complement factor B & $2.82 \mathrm{E}-05$ & 40.22 & $85,478.6$ & 6 \\
\hline DEF3_HUMAN Neutrophil defensin 3 & $6.01 \mathrm{E}-05$ & 10.14 & $10,238.2$ & 2 \\
\hline HEP2_HUMAN Heparin cofactor 2 & $2.17 \mathrm{E}-04$ & 10.25 & $57,034.3$ & 1 \\
\hline \multicolumn{5}{|l|}{ Fraction 23 proteins } \\
\hline K2C6C_HUMAN Keratin, type II cytoskeletal 6C & $1.55 \mathrm{E}-14$ & 10.35 & $59,988.4$ & 3 \\
\hline FIBA_HUMAN Fibrinogen alpha chain & $2.36 \mathrm{E}-11$ & 180.27 & $94,914.3$ & 23 \\
\hline K2C1_HUMAN Keratin, type II cytoskeletal 1 & $4.97 \mathrm{E}-11$ & 110.35 & $65,977.9$ & 12 \\
\hline IBP6_HUMAN Insulin-like growth factor-binding protein 6 & $6.63 \mathrm{E}-11$ & 20.26 & $25,306.2$ & 3 \\
\hline K2C5_HUMAN Keratin, type II cytoskeletal 5 & $2.42 \mathrm{E}-10$ & 10.31 & $62,340.0$ & 1 \\
\hline K1C9_HUMAN Keratin, type I cytoskeletal 9 & $2.71 \mathrm{E}-10$ & 110.33 & $62,091.8$ & 12 \\
\hline K1C10_HUMAN Keratin, type I cytoskeletal 10 & $2.05 \mathrm{E}-09$ & 50.30 & $59,474.8$ & 5 \\
\hline APOA1_HUMAN Apolipoprotein A-I & $7.38 \mathrm{E}-08$ & 100.23 & $30,758.9$ & 31 \\
\hline TTHY_HUMAN Transthyretin & $1.41 \mathrm{E}-07$ & 40.28 & $15,877.1$ & 8 \\
\hline APOC3_HUMAN Apolipoprotein C-III & $7.99 \mathrm{E}-07$ & 10.25 & $10,845.5$ & 1 \\
\hline
\end{tabular}


Table 1 (continued)

\begin{tabular}{|c|c|c|c|c|}
\hline & $\mathrm{P}$ (pro) & Score & MW & Hits \\
\hline CLUS_HUMAN Clusterin & $9.57 \mathrm{E}-07$ & 30.20 & $52,461.1$ & 5 \\
\hline K22E_HUMAN Keratin, type II cytoskeletal 2 epidermal & $1.13 \mathrm{E}-06$ & 10.21 & $65,825.4$ & 1 \\
\hline CFAB_HUMAN Complement factor B & $1.29 \mathrm{E}-06$ & 60.23 & $85,478.6$ & 10 \\
\hline PLMN_HUMAN Plasminogen & $1.69 \mathrm{E}-06$ & 10.25 & $90,510.2$ & 1 \\
\hline K2C75_HUMAN Keratin, type II cytoskeletal 75 & $1.01 \mathrm{E}-04$ & 16.21 & $59,468.0$ & 2 \\
\hline ACTS_HUMAN Actin, alpha skeletal muscle & $1.21 \mathrm{E}-04$ & 10.15 & $42,023.9$ & 1 \\
\hline STP2_HUMAN Nuclear transition protein 2 & $5.46 \mathrm{E}-04$ & 10.19 & $15,630.8$ & 1 \\
\hline \multicolumn{5}{|l|}{ Fraction 24 proteins } \\
\hline K2C6C_HUMAN Keratin, type II cytoskeletal 6C & $1.00 \mathrm{E}-30$ & 10.33 & $59,988.4$ & 2 \\
\hline K22E_HUMAN Keratin, type II cytoskeletal 2 epidermal & $2.22 \mathrm{E}-15$ & 40.25 & $65,825.4$ & 4 \\
\hline K2C1_HUMAN Keratin, type II cytoskeletal 1 & $8.66 \mathrm{E}-14$ & 170.28 & $65,977.9$ & 18 \\
\hline K1C9_HUMAN Keratin, type I cytoskeletal 9 & $5.47 \mathrm{E}-12$ & 80.34 & $62,091.8$ & 9 \\
\hline FIBA_HUMAN Fibrinogen alpha chain & $4.36 \mathrm{E}-10$ & 410.31 & $94,914.3$ & 93 \\
\hline IBP6_HUMAN Insulin-like growth factor-binding protein 6 & $1.62 \mathrm{E}-09$ & 10.24 & $25,306.2$ & 2 \\
\hline APOC3_HUMAN Apolipoprotein C-III & $2.04 \mathrm{E}-09$ & 20.27 & $10,845.5$ & 4 \\
\hline FHR4_HUMAN Complement factor H-related protein 4 & $2.82 \mathrm{E}-09$ & 20.28 & $37,300.4$ & 10 \\
\hline FINC_HUMAN Fibronectin & $7.99 \mathrm{E}-09$ & 110.25 & $262,439.5$ & 15 \\
\hline CO3_HUMAN Complement C3 & $1.44 \mathrm{E}-08$ & 30.23 & $187,029.3$ & 3 \\
\hline KNG1_HUMAN Kininogen-1 & $1.81 \mathrm{E}-08$ & 10.21 & $71,912.1$ & 3 \\
\hline AHNK_HUMAN Neuroblast differentiation-associated protein AHNAK & $2.21 \mathrm{E}-08$ & 144.21 & $628,705.2$ & 17 \\
\hline CFAI_HUMAN Complement factor I & $3.09 \mathrm{E}-08$ & 10.22 & $65,676.7$ & 1 \\
\hline TTHY_HUMAN Transthyretin & $3.45 \mathrm{E}-08$ & 70.27 & $15,877.1$ & 13 \\
\hline PLMN_HUMAN Plasminogen & $6.87 \mathrm{E}-08$ & 20.26 & $90,510.2$ & 3 \\
\hline CLUS_HUMAN Clusterin & $1.57 \mathrm{E}-07$ & 10.21 & $52,461.1$ & 1 \\
\hline EMIL2_HUMAN EMILIN-2 & $1.82 \mathrm{E}-07$ & 10.23 & $115,544.0$ & 1 \\
\hline UPAR_HUMAN Urokinase plasminogen activator surface receptor & $2.83 \mathrm{E}-07$ & 10.25 & $36,953.3$ & 1 \\
\hline APOA1_HUMAN Apolipoprotein A-I & $6.53 \mathrm{E}-07$ & 100.23 & $30,758.9$ & 23 \\
\hline PRIO_HUMAN Major prion protein & $7.31 \mathrm{E}-07$ & 10.21 & $27,643.2$ & 2 \\
\hline HRG_HUMAN Histidine-rich glycoprotein & $1.84 \mathrm{E}-06$ & 10.19 & $59,540.9$ & 1 \\
\hline FHR1_HUMAN Complement factor H-related protein 1 & $2.60 \mathrm{E}-06$ & 30.17 & $37,637.0$ & 3 \\
\hline CFAB_HUMAN Complement factor B & $3.71 \mathrm{E}-06$ & 80.21 & $85,478.6$ & 10 \\
\hline HPT_HUMAN Haptoglobin & $6.41 \mathrm{E}-06$ & 38.18 & $45,176.6$ & 7 \\
\hline FHR3_HUMAN Complement factor H-related protein 3 & $9.61 \mathrm{E}-06$ & 20.16 & $37,298.8$ & 2 \\
\hline K1C10_HUMAN Keratin, type I cytoskeletal 10 & $1.00 \mathrm{E}-05$ & 20.19 & $59,474.8$ & 2 \\
\hline RETBP_PANTR Plasma retinol-binding protein & $1.19 \mathrm{E}-05$ & 30.18 & $22,995.3$ & 4 \\
\hline GDIS_HUMAN Rho GDP-dissociation inhibitor 2 & $1.48 \mathrm{E}-05$ & 20.22 & $22,973.6$ & 2 \\
\hline NHERF_HUMAN Ezrin-radixin-moesin-binding phosphoprotein 50 & $4.74 \mathrm{E}-05$ & 20.16 & $38,844.6$ & 2 \\
\hline IBP3_HUMAN Insulin-like growth factor-binding protein 3 & $9.03 \mathrm{E}-05$ & 40.18 & $31,653.8$ & 7 \\
\hline ACTS_HUMAN Actin, alpha skeletal muscle & $2.25 \mathrm{E}-04$ & 10.15 & $42,023.9$ & 1 \\
\hline K2C75_HUMAN Keratin, type II cytoskeletal 75 & $2.54 \mathrm{E}-04$ & 16.21 & $59,468.0$ & 2 \\
\hline HSPB1_HUMAN Heat shock protein beta-1 & $3.19 \mathrm{E}-04$ & 10.15 & $22,768.5$ & 1 \\
\hline APOC2_HUMAN Apolipoprotein C-II & $4.13 \mathrm{E}-04$ & 10.16 & $11,276.8$ & 1 \\
\hline ADAM8_HUMAN ADAM 8 & $5.40 \mathrm{E}-04$ & 10.15 & $88,615.7$ & 1 \\
\hline \multicolumn{5}{|l|}{ Fraction 30 proteins } \\
\hline HEMO_HUMAN Hemopexin & $5.65 \mathrm{E}-16$ & 604.31 & $51,643.3$ & 307 \\
\hline CFAI_HUMAN Complement factor I & $1.11 \mathrm{E}-15$ & 300.33 & $65,676.7$ & 79 \\
\hline FETUB_HUMAN Fetuin-B & $3.33 \mathrm{E}-15$ & 80.26 & $42,028.0$ & 14 \\
\hline AMBP_HUMAN AMBP protein & $2.00 \mathrm{E}-14$ & 20.24 & $38,974.0$ & 2 \\
\hline IGKC_HUMAN Ig kappa chain C region & $7.99 \mathrm{E}-14$ & 30.27 & $11,601.7$ & 11 \\
\hline
\end{tabular}


Table 1 (continued)

\begin{tabular}{|c|c|c|c|c|}
\hline & $\mathrm{P}$ (pro) & Score & MW & Hits \\
\hline TTHY_HUMAN Transthyretin & $1.11 \mathrm{E}-13$ & 150.29 & $15,877.1$ & 44 \\
\hline TRFE_HUMAN Serotransferrin & $2.02 \mathrm{E}-13$ & 40.33 & $76,999.7$ & 4 \\
\hline RETBP_PANTR Plasma retinol-binding protein & $4.00 \mathrm{E}-13$ & 70.32 & $22,995.3$ & 33 \\
\hline VTDB_HUMAN Vitamin D-binding protein & $9.50 \mathrm{E}-13$ & 20.27 & $52,929.1$ & 3 \\
\hline CO7_HUMAN Complement component C7 & $3.29 \mathrm{E}-12$ & 80.22 & $93,457.3$ & 10 \\
\hline FIBG_HUMAN Fibrinogen gamma chain & $1.25 \mathrm{E}-11$ & 120.29 & $51,478.9$ & 27 \\
\hline FIBA_HUMAN Fibrinogen alpha chain & $1.75 \mathrm{E}-11$ & 70.28 & $94,914.3$ & 8 \\
\hline ITIH4_HUMAN Inter-alpha-trypsin inhibitor heavy chain H4 & $2.39 \mathrm{E}-11$ & 80.29 & $103,261.2$ & 22 \\
\hline ALBU_HUMAN Serum albumin & $4.44 \mathrm{E}-11$ & 716.29 & $69,321.6$ & 473 \\
\hline APOC3_HUMAN Apolipoprotein C-III & $1.08 \mathrm{E}-10$ & 38.26 & $10,845.5$ & 6 \\
\hline FIBB_HUMAN Fibrinogen beta chain & $2.14 \mathrm{E}-10$ & 130.29 & $55,892.2$ & 25 \\
\hline TENX_HUMAN Tenascin-X & $7.70 \mathrm{E}-10$ & 10.21 & $464,165.9$ & 1 \\
\hline A2MG_HUMAN Alpha-2-macroglobulin & $2.49 \mathrm{E}-09$ & 10.17 & $163,174.3$ & 1 \\
\hline HBB_HUMAN Hemoglobin subunit beta & $6.11 \mathrm{E}-09$ & 28.22 & $15,988.3$ & 3 \\
\hline FETUA_HUMAN Alpha-2-HS-glycoprotein & $1.00 \mathrm{E}-08$ & 20.26 & $39,299.7$ & 2 \\
\hline APOA1_HUMAN Apolipoprotein A-I & $2.09 \mathrm{E}-08$ & 110.23 & $30,758.9$ & 34 \\
\hline HPTR_HUMAN Haptoglobin-related protein & $3.73 \mathrm{E}-08$ & 10.18 & $38,982.7$ & 1 \\
\hline K2C1_HUMAN Keratin, type II cytoskeletal 1 & $4.44 \mathrm{E}-08$ & 10.34 & $65,977.9$ & 2 \\
\hline CERU_HUMAN Ceruloplasmin & $4.89 \mathrm{E}-08$ & 10.22 & $122,127.6$ & 1 \\
\hline HBA_HUMAN Hemoglobin subunit alpha & $6.40 \mathrm{E}-08$ & 10.23 & $15,247.9$ & 3 \\
\hline PCOC1_HUMAN Procollagen C-endopeptidase enhancer 1 & $2.70 \mathrm{E}-07$ & 30.16 & $47,942.0$ & 7 \\
\hline CO3A1_HUMAN Collagen alpha-1(III) chain & 4.23E-07 & 20.21 & $138,479.2$ & 2 \\
\hline LAC_HUMAN Ig lambda chain C regions & $5.62 \mathrm{E}-07$ & 20.23 & $11,229.5$ & 9 \\
\hline HPT_HUMAN Haptoglobin & $7.82 \mathrm{E}-07$ & 38.18 & $45,176.6$ & 13 \\
\hline CATB_HUMAN Cathepsin B & $1.21 \mathrm{E}-06$ & 20.21 & $37,796.8$ & 2 \\
\hline MUCB_HUMAN Ig mu heavy chain disease protein & $1.89 \mathrm{E}-06$ & 38.19 & $43,030.3$ & 4 \\
\hline IGHA2_HUMAN Ig alpha-2 chain C region & 4.73E-06 & 10.13 & $36,485.1$ & 2 \\
\hline COR1A_HUMAN Coronin-1A & $6.19 \mathrm{E}-06$ & 10.23 & $50,993.9$ & 1 \\
\hline TETN_HUMAN Tetranectin & $9.66 \mathrm{E}-06$ & 30.24 & $22,552.3$ & 5 \\
\hline IGHM_HUMAN Ig mu chain $\mathrm{C}$ region & $1.93 \mathrm{E}-05$ & 20.26 & $49,275.6$ & 3 \\
\hline CFAB_HUMAN Complement factor B & $2.98 \mathrm{E}-05$ & 40.18 & $85,478.6$ & 4 \\
\hline CAPG_HUMAN Macrophage-capping protein & $3.70 \mathrm{E}-05$ & 40.20 & $38,493.6$ & 6 \\
\hline IGHD_HUMAN Ig delta chain $\mathrm{C}$ region & $4.73 \mathrm{E}-05$ & 10.14 & $42,227.3$ & 1 \\
\hline IGHA1_HUMAN Ig alpha-1 chain C region & $1.52 \mathrm{E}-04$ & 10.18 & $37,630.7$ & 1 \\
\hline ATS9_HUMAN ADAMTS-9 & $4.44 \mathrm{E}-04$ & 10.15 & $216,415.5$ & 1 \\
\hline \multicolumn{5}{|l|}{ Fraction 31 proteins } \\
\hline CFAB_HUMAN Complement factor B & $1.00 \mathrm{E}-30$ & 296.35 & $85,478.6$ & 74 \\
\hline CO8A_HUMAN Complement component C8 alpha chain & $1.00 \mathrm{E}-30$ & 104.27 & $65,121.0$ & 18 \\
\hline TTHY_HUMAN Transthyretin & $1.11 \mathrm{E}-16$ & 340.32 & $15,877.1$ & 103 \\
\hline CERU_HUMAN Ceruloplasmin & $1.11 \mathrm{E}-16$ & 80.31 & $122,127.6$ & 10 \\
\hline RETBP_PANTR Plasma retinol-binding protein & $1.11 \mathrm{E}-16$ & 70.31 & $22,995.3$ & 31 \\
\hline HEMO_HUMAN Hemopexin & $2.22 \mathrm{E}-16$ & 236.31 & $51,643.3$ & 85 \\
\hline K1C9_HUMAN Keratin, type I cytoskeletal 9 & $4.44 \mathrm{E}-16$ & 20.39 & $62,091.8$ & 2 \\
\hline HBA_HUMAN Hemoglobin subunit alpha & $4.44 \mathrm{E}-15$ & 40.29 & $15,247.9$ & 5 \\
\hline S10AC_HUMAN Protein S100-A12 & $6.66 \mathrm{E}-15$ & 10.30 & $10,568.5$ & 1 \\
\hline CO8G_HUMAN Complement component C8 gamma chain & $7.77 \mathrm{E}-15$ & 60.26 & $22,263.6$ & 11 \\
\hline IGKC_HUMAN Ig kappa chain C region & $9.99 \mathrm{E}-15$ & 70.30 & $11,601.7$ & 53 \\
\hline ITIH4_HUMAN Inter-alpha-trypsin inhibitor heavy chain H4 & $1.44 \mathrm{E}-14$ & 468.31 & $103,261.2$ & 188 \\
\hline IGHA1_HUMAN Ig alpha-1 chain C region & $5.46 \mathrm{E}-12$ & 80.32 & $37,630.7$ & 35 \\
\hline
\end{tabular}


Table 1 (continued)

\begin{tabular}{|c|c|c|c|c|}
\hline & $\mathrm{P}$ (pro) & Score & MW & Hits \\
\hline APOC2_HUMAN Apolipoprotein C-II & $5.67 \mathrm{E}-12$ & 60.28 & $11,276.8$ & 14 \\
\hline FIBB_HUMAN Fibrinogen beta & $7.98 \mathrm{E}-12$ & 210.39 & $55,892.2$ & 78 \\
\hline AMBP_HUMAN AMBP protein & $8.25 \mathrm{E}-12$ & 40.25 & $38,974.0$ & 5 \\
\hline CLUS_HUMAN Clusterin & $1.08 \mathrm{E}-11$ & 148.28 & $52,461.1$ & 26 \\
\hline HV3P_HUMAN Ig heavy chain V-III region TEI & $1.38 \mathrm{E}-11$ & 10.28 & $12,794.4$ & 4 \\
\hline ALBU_HUMAN Serum albumin & $1.48 \mathrm{E}-11$ & 370.28 & $69,321.6$ & 160 \\
\hline FIBG_HUMAN Fibrinogen gamma chain & $1.71 \mathrm{E}-11$ & 240.30 & $51,478.9$ & 46 \\
\hline KV1D_HUMAN Ig kappa chain V-I region CAR & $2.53 \mathrm{E}-11$ & 10.26 & $11,696.3$ & 3 \\
\hline AACT_HUMAN Alpha-1-antichymotrypsin & $3.63 \mathrm{E}-11$ & 10.21 & $47,620.6$ & 1 \\
\hline LAC_HUMAN Ig lambda chain $\mathrm{C}$ regions & $5.29 \mathrm{E}-11$ & 60.26 & $11,229.5$ & 16 \\
\hline IGHA2_HUMAN Ig alpha-2 chain $\mathrm{C}$ region & $5.55 \mathrm{E}-11$ & 120.26 & $36,485.1$ & 44 \\
\hline HPT_HUMAN Haptoglobin & $8.17 \mathrm{E}-11$ & 110.29 & $45,176.6$ & 25 \\
\hline ENOB_HUMAN Beta-enolase & $1.28 \mathrm{E}-10$ & 10.24 & $46,957.4$ & 1 \\
\hline ENOA_HUMAN Alpha-enolase & $1.31 \mathrm{E}-10$ & 30.26 & $47,139.4$ & 3 \\
\hline APOA1_HUMAN Apolipoprotein A-I & $1.45 \mathrm{E}-10$ & 330.30 & $30,758.9$ & 111 \\
\hline CFAI_HUMAN Complement factor I & $1.53 \mathrm{E}-10$ & 118.28 & $65,676.7$ & 23 \\
\hline COR1A_HUMAN Coronin-1A & $1.97 \mathrm{E}-10$ & 64.25 & $50,993.9$ & 8 \\
\hline HBD_HUMAN Hemoglobin subunit delta & $2.66 \mathrm{E}-10$ & 78.23 & $16,045.3$ & 12 \\
\hline FETUA_HUMAN Alpha-2-HS-glycoprotein & $6.50 \mathrm{E}-10$ & 20.27 & $39,299.7$ & 4 \\
\hline DIAC_HUMAN Di-N-acetylchitobiase & $9.02 \mathrm{E}-10$ & 20.20 & $43,731.6$ & 2 \\
\hline A2AP_HUMAN Alpha-2-antiplasmin & $9.62 \mathrm{E}-10$ & 40.24 & $54,531.2$ & 4 \\
\hline KV3H_HUMAN Ig kappa chain V-III region CLL & $1.61 \mathrm{E}-09$ & 18.23 & $14,266.2$ & 4 \\
\hline SHBG_HUMAN Sex hormone-binding globulin & $2.19 \mathrm{E}-09$ & 60.24 & $43,751.9$ & 6 \\
\hline ENO1B_HUMAN Alpha-enolase, lung specific & $2.99 \mathrm{E}-09$ & 10.21 & $49,446.4$ & 2 \\
\hline AIF1_HUMAN Allograft inflammatory factor 1 & $3.56 \mathrm{E}-09$ & 10.23 & $16,692.6$ & 1 \\
\hline FRIH_HUMAN Ferritin heavy chain & $5.57 \mathrm{E}-09$ & 60.25 & $21,212.3$ & 8 \\
\hline FIBA_HUMAN Fibrinogen alpha chain & $7.21 \mathrm{E}-09$ & 98.28 & $94,914.3$ & 17 \\
\hline IGHD_HUMAN Ig delta chain $\mathrm{C}$ region & $7.77 \mathrm{E}-09$ & 30.23 & $42,227.3$ & 6 \\
\hline LUM_HUMAN Lumican & $1.22 \mathrm{E}-08$ & 60.30 & $38,404.8$ & 14 \\
\hline GELS_HUMAN Gelsolin & $2.34 \mathrm{E}-08$ & 20.21 & $85,644.3$ & 2 \\
\hline HBB_HUMAN Hemoglobin subunit beta & $2.70 \mathrm{E}-08$ & 50.22 & $15,988.3$ & 14 \\
\hline COF1_HUMAN Cofilin-1 & $3.91 \mathrm{E}-08$ & 10.26 & $18,490.7$ & 1 \\
\hline KV1S_HUMAN Ig kappa chain V-I region Wes & $4.88 \mathrm{E}-08$ & 10.21 & $11,600.7$ & 1 \\
\hline MUCB_HUMAN Ig mu heavy chain disease protein & $5.93 \mathrm{E}-08$ & 56.22 & $43,030.3$ & 15 \\
\hline LV3B_HUMAN Ig lambda chain V-III region LOI & $9.13 \mathrm{E}-08$ & 20.24 & $11,927.8$ & 6 \\
\hline FETUB_HUMAN Fetuin-B & $9.28 \mathrm{E}-08$ & 60.27 & $42,028.0$ & 8 \\
\hline K2C1_HUMAN Keratin, type II cytoskeletal 1 & $1.53 \mathrm{E}-07$ & 46.37 & $65,977.9$ & 6 \\
\hline IGHM_HUMAN Ig mu chain $\mathrm{C}$ region & $2.18 \mathrm{E}-07$ & 30.23 & $49,275.6$ & 5 \\
\hline MMP1_HUMAN Interstitial collagenase & $2.78 \mathrm{E}-07$ & 30.21 & $53,972.8$ & 3 \\
\hline COTL1_HUMAN Coactosin-like protein & $3.14 \mathrm{E}-07$ & 10.23 & $15,935.0$ & 1 \\
\hline KV3G_HUMAN Ig kappa chain V-III region GOL & $3.86 \mathrm{E}-07$ & 10.21 & $11,823.0$ & 4 \\
\hline CATB_HUMAN Cathepsin B & $3.96 \mathrm{E}-07$ & 10.16 & $37,796.8$ & 1 \\
\hline S10A9_HUMAN Protein S100-A9 & $4.68 \mathrm{E}-07$ & 10.19 & $13,233.5$ & 1 \\
\hline THIO_HUMAN Thioredoxin & $4.91 \mathrm{E}-07$ & 10.18 & $11,729.7$ & 4 \\
\hline TRFE_HUMAN Serotransferrin & $5.57 \mathrm{E}-07$ & 50.26 & 136,191 & 6 \\
\hline A2MG_HUMAN Alpha-2-macroglobulin & $6.03 \mathrm{E}-07$ & 20.21 & $163,174.3$ & 3 \\
\hline ANT3_HUMAN Antithrombin-III & $6.29 \mathrm{E}-07$ & 10.21 & $52,569.0$ & 1 \\
\hline KPYM_HUMAN Pyruvate kinase isozymes M1/M2 & $7.06 \mathrm{E}-07$ & 10.24 & $57,900.2$ & 1 \\
\hline PROF1_HUMAN Profilin-1 (Profilin I) & $8.10 \mathrm{E}-07$ & 30.20 & $15,044.6$ & 3 \\
\hline
\end{tabular}


Table 1 (continued)

\begin{tabular}{|c|c|c|c|c|}
\hline & $\mathrm{P}$ (pro) & Score & MW & Hits \\
\hline CO8B_HUMAN Complement component C8 beta chain & $8.76 \mathrm{E}-07$ & 30.17 & $67,003.5$ & 3 \\
\hline PCOC1_HUMAN Procollagen C-endopeptidase enhancer 1 & $1.74 \mathrm{E}-06$ & 20.19 & $47,942.0$ & 2 \\
\hline HV3J_HUMAN Ig heavy chain V-III region HIL & $1.97 \mathrm{E}-06$ & 10.19 & $13,557.2$ & 3 \\
\hline SAMP_HUMAN Serum amyloid P-component & $2.29 \mathrm{E}-06$ & 10.16 & $25,371.1$ & 1 \\
\hline KV3I_HUMAN Ig kappa chain V-III region VG & $2.90 \mathrm{E}-06$ & 20.25 & $12,567.3$ & 4 \\
\hline CAPG_HUMAN Macrophage-capping protein & $3.08 \mathrm{E}-06$ & 10.20 & $38,493.6$ & 1 \\
\hline APOC3_HUMAN Apolipoprotein C-III & $4.71 \mathrm{E}-06$ & 20.21 & $10,845.5$ & 3 \\
\hline CO7_HUMAN Complement component C7 & $5.24 \mathrm{E}-06$ & 48.24 & $93,457.3$ & 6 \\
\hline ADIPO_HUMAN Adiponectin & $7.98 \mathrm{E}-06$ & 10.22 & $26,397.0$ & 1 \\
\hline KV1V_HUMAN Ig kappa chain V-I region BAN & $9.70 \mathrm{E}-06$ & 10.21 & $11,832.8$ & 1 \\
\hline HV1G_HUMAN Ig heavy chain V-I region V35 & $1.01 \mathrm{E}-05$ & 10.15 & $13,000.4$ & 1 \\
\hline KV4C_HUMAN Ig kappa chain V-IV region B17 & $2.11 \mathrm{E}-05$ & 20.16 & $14,956.5$ & 2 \\
\hline TETN_HUMAN Tetranectin & $2.36 \mathrm{E}-05$ & 10.18 & $22,552.3$ & 1 \\
\hline APOD_HUMAN Apolipoprotein D & $7.83 \mathrm{E}-05$ & 20.13 & $21,261.8$ & 4 \\
\hline IGLL1_HUMAN Immunoglobulin lambda-like polypeptide 1 & $8.60 \mathrm{E}-05$ & 28.19 & $22,948.6$ & 3 \\
\hline IGJ_HUMAN Immunoglobulin J chain & $8.94 \mathrm{E}-05$ & 10.13 & $15,584.6$ & 1 \\
\hline HV3R_HUMAN Ig heavy chain V-III region TUR & $1.01 \mathrm{E}-04$ & 10.22 & $12,423.3$ & 3 \\
\hline SAA4_HUMAN Serum amyloid A-4 protein & $1.78 \mathrm{E}-04$ & 10.16 & $14,797.3$ & 2 \\
\hline HV1C_HUMAN Ig heavy chain V-I region ND & $1.93 \mathrm{E}-04$ & 10.13 & $16,493.0$ & 1 \\
\hline HPTR_HUMAN Haptoglobin-related protein & $1.94 \mathrm{E}-04$ & 10.18 & $38,982.7$ & 1 \\
\hline LV3A_HUMAN Ig lambda chain V-III region SH & $5.30 \mathrm{E}-04$ & 10.17 & $11,385.6$ & 2 \\
\hline CO1A1_HUMAN Collagen alpha-1(I) chain & $5.86 \mathrm{E}-04$ & 10.15 & $138,826.8$ & 1 \\
\hline MBOA2_HUMAN Membrane-bound $O$-acyltransferase domain-containing & $7.03 \mathrm{E}-04$ & 10.11 & $59,488.3$ & 1 \\
\hline ZA2G_HUMAN Zinc-alpha-2-glycoprotein & $8.67 \mathrm{E}-04$ & 10.14 & $33,850.9$ & 1 \\
\hline \multicolumn{5}{|l|}{ Fraction 32 proteins } \\
\hline TTHY_HUMAN Transthyretin & $1.00 \mathrm{E}-30$ & 350.34 & $15,877.1$ & 114 \\
\hline HBA_HUMAN Hemoglobin subunit alpha & $1.00 \mathrm{E}-30$ & 90.31 & $15,247.9$ & 15 \\
\hline CO8B_HUMAN Complement component C8 beta chain & $1.00 \mathrm{E}-30$ & 10.26 & $67,003.5$ & 1 \\
\hline CFAB_HUMAN Complement factor B & $7.77 \mathrm{E}-15$ & 220.34 & $85,478.6$ & 52 \\
\hline ITIH2_HUMAN Inter-alpha-trypsin inhibitor heavy chain H2 & $8.88 \mathrm{E}-15$ & 10.29 & $106,369.8$ & 1 \\
\hline CO8G_HUMAN Complement component C8 gamma chain & $9.99 \mathrm{E}-15$ & 30.25 & $22,263.6$ & 5 \\
\hline K1C9_HUMAN Keratin, type I cytoskeletal 9 & $9.99 \mathrm{E}-15$ & 20.38 & $62,091.8$ & 2 \\
\hline CERU_HUMAN Ceruloplasmin & $1.11 \mathrm{E}-14$ & 60.28 & $122,127.6$ & 7 \\
\hline CLUS_HUMAN Clusterin & $1.67 \mathrm{E}-14$ & 190.29 & $52,461.1$ & 57 \\
\hline G3P_HUMAN Glyceraldehyde-3-phosphate dehydrogenase & $2.11 \mathrm{E}-14$ & 50.33 & $36,030.4$ & 5 \\
\hline CBPN_HUMAN Carboxypeptidase N catalytic chain & $3.55 \mathrm{E}-14$ & 20.26 & $52,253.4$ & 3 \\
\hline RETBP_PANTR Plasma retinol-binding protein & $4.00 \mathrm{E}-14$ & 60.32 & $22,995.3$ & 34 \\
\hline AMBP_HUMAN AMBP protein & $4.00 \mathrm{E}-14$ & 30.27 & $38,974.0$ & 4 \\
\hline ENOA_HUMAN Alpha-enolase & $5.33 \mathrm{E}-14$ & 148.35 & $47,139.4$ & 43 \\
\hline IGKC_HUMAN Ig kappa chain $\mathrm{C}$ region & $5.77 \mathrm{E}-14$ & 60.29 & $11,601.7$ & 66 \\
\hline HEMO_HUMAN Hemopexin & $2.15 \mathrm{E}-13$ & 136.31 & $51,643.3$ & 41 \\
\hline ANT3_HUMAN Antithrombin-III & $7.48 \mathrm{E}-13$ & 316.31 & $52,569.0$ & 59 \\
\hline S10AC_HUMAN Protein S100-A12 & $1.80 \mathrm{E}-12$ & 40.36 & $10,568.5$ & 8 \\
\hline HBD_HUMAN Hemoglobin subunit delta & $2.46 \mathrm{E}-12$ & 206.30 & $16,045.3$ & 77 \\
\hline KLKB1_HUMAN Plasma kallikrein & $2.60 \mathrm{E}-12$ & 50.24 & $71,322.8$ & 6 \\
\hline ENOB_HUMAN Beta-enolase & $2.71 \mathrm{E}-12$ & 38.26 & $46,957.4$ & 9 \\
\hline SHBG_HUMAN Sex hormone-binding globulin & $5.08 \mathrm{E}-12$ & 90.29 & $43,751.9$ & 23 \\
\hline ITIH4_HUMAN Inter-alpha-trypsin inhibitor heavy chain H4 & $6.78 \mathrm{E}-12$ & 110.29 & $103,261.2$ & 25 \\
\hline S10A8_HUMAN Protein S100-A8 & $7.06 \mathrm{E}-12$ & 20.31 & $10,827.7$ & 5 \\
\hline
\end{tabular}


Table 1 (continued)

\begin{tabular}{|c|c|c|c|c|}
\hline & $P($ pro $)$ & Score & MW & Hits \\
\hline APOC2_HUMAN Apolipoprotein C-II & $9.10 \mathrm{E}-12$ & 100.29 & $11,276.8$ & 30 \\
\hline PGRP2_HUMAN N-acetylmuramoyl-L-alanine amidase & $1.26 \mathrm{E}-11$ & 60.29 & $62,178.0$ & 8 \\
\hline KV1D_HUMAN Ig kappa chain V-I region CAR & $1.76 \mathrm{E}-11$ & 10.25 & $11,696.3$ & 3 \\
\hline KV1S_HUMAN Ig kappa chain V-I region Wes & $2.20 \mathrm{E}-11$ & 10.23 & $11,600.7$ & 2 \\
\hline APOM_HUMAN Apolipoprotein M & $2.21 \mathrm{E}-11$ & 70.25 & $21,239.4$ & 11 \\
\hline HBB_HUMAN Hemoglobin subunit beta & $2.64 \mathrm{E}-11$ & 208.31 & $15,988.3$ & 131 \\
\hline IGHA1_HUMAN Ig alpha-1 chain C region & $3.97 \mathrm{E}-11$ & 80.26 & $37,630.7$ & 29 \\
\hline LAC_HUMAN Ig lambda chain C regions & $6.32 \mathrm{E}-11$ & 60.27 & $11,229.5$ & 19 \\
\hline CFAI_HUMAN Complement factor I & $6.39 \mathrm{E}-11$ & 116.28 & $65,676.7$ & 21 \\
\hline KV4C_HUMAN Ig kappa chain V-IV region B17 & $7.16 \mathrm{E}-11$ & 20.26 & $14,956.5$ & 3 \\
\hline THRB_HUMAN Prothrombin & $1.36 \mathrm{E}-10$ & 40.28 & $69,992.2$ & 5 \\
\hline APOA1_HUMAN Apolipoprotein A-I & $1.44 \mathrm{E}-10$ & 530.26 & $30,758.9$ & 302 \\
\hline CFAD_HUMAN Complement factor D & $2.12 \mathrm{E}-10$ & 30.22 & $27,015.9$ & 4 \\
\hline IGHA2_HUMAN Ig alpha-2 chain $\mathrm{C}$ region & $2.79 \mathrm{E}-10$ & 118.23 & $36,485.1$ & 36 \\
\hline FIBB_HUMAN Fibrinogen beta chain & $2.86 \mathrm{E}-10$ & 100.27 & $55,892.2$ & 16 \\
\hline HPT_HUMAN Haptoglobin & $3.59 \mathrm{E}-10$ & 130.32 & $45,176.6$ & 28 \\
\hline SAMP_HUMAN Serum amyloid P-component & $4.06 \mathrm{E}-10$ & 60.21 & $25,371.1$ & 12 \\
\hline KV3H_HUMAN Ig kappa chain V-III region CLL & $5.94 \mathrm{E}-10$ & 10.22 & $14,266.2$ & 2 \\
\hline APOA2_HUMAN Apolipoprotein A-II & $7.84 \mathrm{E}-10$ & 60.31 & $11,167.9$ & 7 \\
\hline HSP71_PONAB Heat shock 70 kDa protein 1 & $8.30 \mathrm{E}-10$ & 10.24 & $70,009.2$ & 1 \\
\hline CO8A_HUMAN Complement component C8 alpha chain & $9.20 \mathrm{E}-10$ & 20.25 & $65,121.0$ & 2 \\
\hline S10A9_HUMAN Protein S100-A9 & $1.43 \mathrm{E}-09$ & 40.25 & $13,233.5$ & 7 \\
\hline ALBU_HUMAN Serum albumin & $2.15 \mathrm{E}-09$ & 262.26 & $69,321.6$ & 70 \\
\hline VASP_HUMAN Vasodilator-stimulated phosphoprotein & $2.32 \mathrm{E}-09$ & 10.23 & $39,805.1$ & 1 \\
\hline K2C1_HUMAN Keratin, type II cytoskeletal 1 & $2.40 \mathrm{E}-09$ & 26.41 & $65,977.9$ & 4 \\
\hline FIBA_HUMAN Fibrinogen alpha chain & $2.63 \mathrm{E}-09$ & 30.23 & $94,914.3$ & 3 \\
\hline ADIPO_HUMAN Adiponectin & $3.36 \mathrm{E}-09$ & 10.22 & $26,397.0$ & 1 \\
\hline FIBG_HUMAN Fibrinogen gamma chain & $1.33 \mathrm{E}-08$ & 70.27 & $51,478.9$ & 14 \\
\hline ENO1B_HUMAN Alpha-enolase, lung specific & $1.53 \mathrm{E}-08$ & 50.29 & $49,446.4$ & 9 \\
\hline LV4C_HUMAN Ig lambda chain V-IV region Hil & $1.59 \mathrm{E}-08$ & 10.25 & $11,509.6$ & 2 \\
\hline IGHG3_HUMAN Ig gamma-3 chain C region & $2.06 \mathrm{E}-08$ & 10.18 & $41,260.4$ & 1 \\
\hline COR1A_HUMAN Coronin-1A & $2.58 \mathrm{E}-08$ & 10.24 & $50,993.9$ & 1 \\
\hline CO4A_HUMAN Complement C4-A & $3.08 \mathrm{E}-08$ & 20.24 & $192,649.5$ & 2 \\
\hline KV3G_HUMAN Ig kappa chain V-III region GOL & $3.61 \mathrm{E}-08$ & 10.21 & $11,823.0$ & 3 \\
\hline IGHD_HUMAN Ig delta chain C region & $3.83 \mathrm{E}-08$ & 20.19 & $42,227.3$ & 3 \\
\hline FRIH_HUMAN Ferritin heavy chain & $3.91 \mathrm{E}-08$ & 10.21 & $21,212.3$ & 1 \\
\hline IGHM_HUMAN Ig mu chain C region & $5.37 \mathrm{E}-08$ & 20.23 & $49,275.6$ & 13 \\
\hline LUM_HUMAN Lumican & $5.61 \mathrm{E}-08$ & 80.26 & $38,404.8$ & 17 \\
\hline PSA6_RAT Proteasome subunit alpha type-6 & $6.04 \mathrm{E}-08$ & 20.17 & $27,381.8$ & 2 \\
\hline MUCB_HUMAN Ig mu heavy chain disease protein & $1.04 \mathrm{E}-07$ & 58.23 & $43,030.3$ & 12 \\
\hline LV3B_HUMAN Ig lambda chain V-III region LOI & $1.17 \mathrm{E}-07$ & 30.24 & $11,927.8$ & 6 \\
\hline CO1A1_HUMAN Collagen alpha-1(I) chain & $1.76 \mathrm{E}-07$ & 10.15 & $138,826.8$ & 1 \\
\hline APOE_HUMAN Apolipoprotein E & $1.87 \mathrm{E}-07$ & 20.19 & $36,131.8$ & 3 \\
\hline CAPG_HUMAN Macrophage-capping protein & $2.00 \mathrm{E}-07$ & 10.19 & $38,493.6$ & 1 \\
\hline LMNA_HUMAN Lamin-A/C & $2.15 \mathrm{E}-07$ & 30.20 & $74,094.8$ & 4 \\
\hline A2MG_HUMAN Alpha-2-macroglobulin & $3.81 \mathrm{E}-07$ & 60.19 & $163,174.3$ & 7 \\
\hline IGLL1_HUMAN Immunoglobulin lambda-like polypeptide 1 & $4.24 \mathrm{E}-07$ & 38.22 & $22,948.6$ & 5 \\
\hline CFAH_HUMAN Complement factor $\mathrm{H}$ & $6.11 \mathrm{E}-07$ & 10.14 & $139,004.4$ & 1 \\
\hline GELS_HUMAN Gelsolin & $1.31 \mathrm{E}-06$ & 20.23 & $85,644.3$ & 2 \\
\hline
\end{tabular}


Table 1 (continued)

\begin{tabular}{|c|c|c|c|c|}
\hline & $\mathrm{P}$ (pro) & Score & MW & Hits \\
\hline HV1G_HUMAN Ig heavy chain V-I region V35 & $1.50 \mathrm{E}-06$ & 10.16 & $13,000.4$ & 1 \\
\hline CO3_HUMAN Complement C3 & $1.59 \mathrm{E}-06$ & 30.22 & $187,029.3$ & 3 \\
\hline FETUB_HUMAN Fetuin-B & $1.64 \mathrm{E}-06$ & 10.26 & $42,028.0$ & 1 \\
\hline UBP15_HUMAN Ubiquitin carboxyl-terminal hydrolase 15 & $3.10 \mathrm{E}-06$ & 10.20 & $112,347.6$ & 1 \\
\hline GPX3_HUMAN Glutathione peroxidase 3 & $3.23 \mathrm{E}-06$ & 60.20 & $25,386.0$ & 6 \\
\hline FETUA_HUMAN Alpha-2-HS-glycoprotein & $4.85 \mathrm{E}-06$ & 20.18 & $39,299.7$ & 2 \\
\hline APOA4_HUMAN Apolipoprotein A-IV & $5.76 \mathrm{E}-06$ & 10.19 & $45,371.5$ & 1 \\
\hline FGFP2_HUMAN Fibroblast growth factor-binding protein 2 & $1.29 \mathrm{E}-05$ & 20.18 & $24,565.2$ & 2 \\
\hline APOC1_HUMAN Apolipoprotein C-I & $2.47 \mathrm{E}-05$ & 10.12 & $9,326.1$ & 1 \\
\hline APOD_HUMAN Apolipoprotein D & $2.86 \mathrm{E}-05$ & 10.12 & $21,261.8$ & 1 \\
\hline HV3P_HUMAN Ig heavy chain V-III region TEI & $2.88 \mathrm{E}-05$ & 10.20 & $12,794.4$ & 3 \\
\hline GDIS_HUMAN Rho GDP-dissociation inhibitor 2 & $3.11 \mathrm{E}-05$ & 10.18 & $22,973.6$ & 1 \\
\hline TENA_HUMAN Tenascin & $3.65 \mathrm{E}-05$ & 10.16 & $240,710.7$ & 1 \\
\hline HV1B_HUMAN Ig heavy chain V-I region HG3 & $3.81 \mathrm{E}-05$ & 10.17 & $12,937.3$ & 1 \\
\hline TRFE_HUMAN Serotransferrin & $4.38 \mathrm{E}-05$ & 66.22 & $76,999.7$ & 7 \\
\hline PROF1_HUMAN Profilin-1 & $4.39 \mathrm{E}-05$ & 10.22 & $15,044.6$ & 1 \\
\hline KV3I_HUMAN Ig kappa chain V-III region VG & $8.00 \mathrm{E}-05$ & 20.25 & $12,567.3$ & 3 \\
\hline APOB_HUMAN Apolipoprotein B-100 & $8.41 \mathrm{E}-05$ & 20.21 & $515,241.6$ & 2 \\
\hline LV3A_HUMAN Ig lambda chain V-III region SH & $8.52 \mathrm{E}-05$ & 10.21 & $11,385.6$ & 1 \\
\hline APOC3_HUMAN Apolipoprotein C-III & $8.88 \mathrm{E}-05$ & 10.16 & $10,845.5$ & 1 \\
\hline PRDX6_HUMAN Peroxiredoxin-6 & $9.49 \mathrm{E}-05$ & 10.14 & $25,019.2$ & 1 \\
\hline DOPO_HUMAN Dopamine beta-hydroxylase & $1.01 \mathrm{E}-04$ & 20.16 & $69,020.8$ & 2 \\
\hline KV1V_HUMAN Ig kappa chain V-I region BAN & $1.47 \mathrm{E}-04$ & 10.19 & $11,832.8$ & 2 \\
\hline TRIM7_HUMAN Tripartite motif-containing protein 7 & $2.81 \mathrm{E}-04$ & 10.15 & $56,595.0$ & 6 \\
\hline SAA4_HUMAN Serum amyloid A-4 protein & $3.43 \mathrm{E}-04$ & 20.16 & $14,797.3$ & 4 \\
\hline \multicolumn{5}{|l|}{ Fraction 33 proteins } \\
\hline HBA_HUMAN Hemoglobin subunit alpha & $1.00 \mathrm{E}-30$ & 350.39 & $15,247.9$ & 127 \\
\hline PGRP2_HUMAN N-acetylmuramoyl-L-alanine amidase & $1.11 \mathrm{E}-15$ & 268.33 & $62,178.0$ & 53 \\
\hline CFAB_HUMAN Complement factor B & $1.11 \mathrm{E}-15$ & 180.34 & $85,478.6$ & 30 \\
\hline RETBP_PANTR Plasma retinol-binding protein & $1.11 \mathrm{E}-15$ & 70.32 & $22,995.3$ & 30 \\
\hline APOA1_HUMAN Apolipoprotein A-I & $8.88 \mathrm{E}-15$ & 648.34 & $30,758.9$ & 410 \\
\hline S10AC_HUMAN Protein S100-A12 & $1.22 \mathrm{E}-14$ & 20.35 & $10,568.5$ & 3 \\
\hline CO3_HUMAN Complement C3 & $2.11 \mathrm{E}-14$ & 468.34 & $187,029.3$ & 76 \\
\hline HEMO_HUMAN Hemopexin & $6.33 \mathrm{E}-14$ & 178.29 & $51,643.3$ & 30 \\
\hline TTHY_HUMAN Transthyretin & $1.45 \mathrm{E}-13$ & 220.33 & $15,877.1$ & 49 \\
\hline CFAI_HUMAN Complement factor I & $2.96 \mathrm{E}-13$ & 88.28 & $65,676.7$ & 14 \\
\hline ENOA_HUMAN Alpha-enolase & $3.60 \mathrm{E}-13$ & 50.33 & $47,139.4$ & 7 \\
\hline CERU_HUMAN Ceruloplasmin & $3.69 \mathrm{E}-13$ & 60.33 & $122,127.6$ & 6 \\
\hline K2C1_HUMAN Keratin, type II cytoskeletal 1 & $3.94 \mathrm{E}-13$ & 76.34 & $65,977.9$ & 9 \\
\hline ITIH2_HUMAN Inter-alpha-trypsin inhibitor heavy chain H2 & $1.32 \mathrm{E}-12$ & 50.33 & $106,369.8$ & 6 \\
\hline ENOB_HUMAN Beta-enolase & $1.48 \mathrm{E}-12$ & 10.25 & $46,957.4$ & 3 \\
\hline CO8G_HUMAN Complement component C8 gamma chain & $3.98 \mathrm{E}-12$ & 20.20 & $22,263.6$ & 3 \\
\hline ANT3_HUMAN Antithrombin-III & $4.03 \mathrm{E}-12$ & 268.30 & $52,569.0$ & 39 \\
\hline IGKC_HUMAN Ig kappa chain $\mathrm{C}$ region & $4.14 \mathrm{E}-12$ & 100.29 & $11,601.7$ & 54 \\
\hline IGHA1_HUMAN Ig alpha-1 chain C region & $1.07 \mathrm{E}-11$ & 90.29 & $37,630.7$ & 32 \\
\hline KV1D_HUMAN Ig kappa chain V-I region CAR & $1.20 \mathrm{E}-11$ & 20.26 & $11,696.3$ & 3 \\
\hline APOM_HUMAN Apolipoprotein M & $1.29 \mathrm{E}-11$ & 60.25 & $21,239.4$ & 8 \\
\hline CO4A_HUMAN Complement C4-A & $1.48 \mathrm{E}-11$ & 200.28 & $192,649.5$ & 25 \\
\hline PSA2_HUMAN Proteasome subunit alpha type-2 & $1.55 \mathrm{E}-11$ & 60.29 & $25,882.3$ & 8 \\
\hline
\end{tabular}


Table 1 (continued)

\begin{tabular}{|c|c|c|c|c|}
\hline & $P($ pro $)$ & Score & MW & Hits \\
\hline HV3R_HUMAN Ig heavy chain V-III region TUR & $2.17 \mathrm{E}-11$ & 20.25 & $12,423.3$ & 4 \\
\hline KV1R_HUMAN Ig kappa chain V-I region WEA & $2.35 \mathrm{E}-11$ & 26.28 & $11,832.8$ & 8 \\
\hline HBB_HUMAN Hemoglobin subunit beta & $3.15 \mathrm{E}-11$ & 190.27 & $15,988.3$ & 85 \\
\hline HBD_HUMAN Hemoglobin subunit delta & $4.14 \mathrm{E}-11$ & 228.29 & $16,045.3$ & 66 \\
\hline GSTP1_HUMAN Glutathione $S$-transferase P & $7.69 \mathrm{E}-11$ & 30.25 & $23,341.0$ & 4 \\
\hline LV4C_HUMAN Ig lambda chain V-IV region Hil & $8.74 \mathrm{E}-11$ & 20.26 & $11,509.6$ & 4 \\
\hline FIBA_HUMAN Fibrinogen alpha chain & $9.51 \mathrm{E}-11$ & 20.26 & $94,914.3$ & 2 \\
\hline APOC2_HUMAN Apolipoprotein C-II & $1.01 \mathrm{E}-10$ & 100.28 & $11,276.8$ & 17 \\
\hline LAC_HUMAN Ig lambda chain C regions & $1.09 \mathrm{E}-10$ & 50.27 & $11,229.5$ & 14 \\
\hline S10A9_HUMAN Protein S100-A9 & $1.16 \mathrm{E}-10$ & 128.28 & $13,233.5$ & 31 \\
\hline CLUS_HUMAN Clusterin & $2.11 \mathrm{E}-10$ & 218.30 & $52,461.1$ & 47 \\
\hline SAMP_HUMAN Serum amyloid P-component & $2.58 \mathrm{E}-10$ & 10.19 & $25,371.1$ & 2 \\
\hline HV3P_HUMAN Ig heavy chain V-III region TEI & $4.78 \mathrm{E}-10$ & 10.26 & $12,794.4$ & 3 \\
\hline KV3H_HUMAN Ig kappa chain V-III region CLL & $6.52 \mathrm{E}-10$ & 10.21 & $14,266.2$ & 2 \\
\hline PERM_HUMAN Myeloperoxidase & $6.57 \mathrm{E}-10$ & 10.21 & $83,815.0$ & 1 \\
\hline ALBU_HUMAN Serum albumin & $6.70 \mathrm{E}-10$ & 190.27 & $69,321.6$ & 44 \\
\hline SPB10_HUMAN Serpin B10 & $7.84 \mathrm{E}-10$ & 30.21 & $45,374.0$ & 3 \\
\hline CO6A1_HUMAN Collagen alpha-1(VI) chain & $7.93 \mathrm{E}-10$ & 20.26 & $108,462.0$ & 3 \\
\hline A2GL_HUMAN Leucine-rich alpha-2-glycoprotein & $8.29 \mathrm{E}-10$ & 50.25 & $38,154.1$ & 8 \\
\hline IGHA2_HUMAN Ig alpha-2 chain C region & $1.05 \mathrm{E}-09$ & 94.24 & $36,485.1$ & 28 \\
\hline ITIH1_HUMAN Inter-alpha-trypsin inhibitor heavy chain H1 & $1.10 \mathrm{E}-09$ & 30.26 & $101,325.8$ & 3 \\
\hline KV1S_HUMAN Ig kappa chain V-I region Wes & $1.21 \mathrm{E}-09$ & 10.23 & $11,600.7$ & 1 \\
\hline APOA2_HUMAN Apolipoprotein A-II & $1.22 \mathrm{E}-09$ & 100.28 & $11,167.9$ & 13 \\
\hline CATD_HUMAN Cathepsin D & $1.30 \mathrm{E}-09$ & 32.22 & $44,523.7$ & 4 \\
\hline A2MG_HUMAN Alpha-2-macroglobulin & $1.34 \mathrm{E}-09$ & 120.23 & $163,174.3$ & 16 \\
\hline SHBG_HUMAN Sex hormone-binding globulin & $1.66 \mathrm{E}-09$ & 10.29 & $43,751.9$ & 1 \\
\hline KV4C_HUMAN Ig kappa chain V-IV region B17 & $1.69 \mathrm{E}-09$ & 30.24 & $14,956.5$ & 5 \\
\hline TRFE_HUMAN Serotransferrin & $4.52 \mathrm{E}-09$ & 20.21 & $76,999.7$ & 2 \\
\hline ITIH4_HUMAN Inter-alpha-trypsin inhibitor heavy chain H4 & $6.22 \mathrm{E}-09$ & 110.26 & $103,261.2$ & 16 \\
\hline MUCB_HUMAN Ig mu heavy chain disease protein & $6.34 \mathrm{E}-09$ & 68.25 & $43,030.3$ & 20 \\
\hline S10A8_HUMAN Protein S100-A8 & $6.64 \mathrm{E}-09$ & 20.25 & $10,827.7$ & 2 \\
\hline HSP71_PONAB Heat shock 70 kDa protein 1 & $6.79 \mathrm{E}-09$ & 20.27 & $70,009.2$ & 2 \\
\hline HPT_HUMAN Haptoglobin & $7.42 \mathrm{E}-09$ & 132.26 & $45,176.6$ & 24 \\
\hline KV3G_HUMAN Ig kappa chain V-III region GOL & $7.50 \mathrm{E}-09$ & 10.23 & $11,823.0$ & 2 \\
\hline AFAM_HUMAN Afamin & $2.07 \mathrm{E}-08$ & 70.24 & $69,024.1$ & 7 \\
\hline FIBB_HUMAN Fibrinogen beta chain & $2.09 \mathrm{E}-08$ & 70.24 & $55,892.2$ & 8 \\
\hline K1C9_HUMAN Keratin, type I cytoskeletal 9 & $2.09 \mathrm{E}-08$ & 40.33 & $62,091.8$ & 4 \\
\hline TKT_HUMAN Transketolase & $2.24 \mathrm{E}-08$ & 40.21 & $67,834.9$ & 5 \\
\hline IGHM_HUMAN Ig mu chain C region & $2.76 \mathrm{E}-08$ & 50.25 & $49,275.6$ & 9 \\
\hline GELS_HUMAN Gelsolin & $3.10 \mathrm{E}-08$ & 10.23 & $85,644.3$ & 1 \\
\hline FIBG_HUMAN Fibrinogen gamma chain & $3.14 \mathrm{E}-08$ & 50.19 & $51,478.9$ & 5 \\
\hline IGHD_HUMAN Ig delta chain C region & $3.27 \mathrm{E}-08$ & 20.19 & $42,227.3$ & 3 \\
\hline ADIPO_HUMAN Adiponectin & $4.87 \mathrm{E}-08$ & 10.23 & $26,397.0$ & 1 \\
\hline PRDX2_HUMAN Peroxiredoxin-2 & $5.29 \mathrm{E}-08$ & 50.21 & $21,878.2$ & 6 \\
\hline LV3B_HUMAN Ig lambda chain V-III region LOI & $7.16 \mathrm{E}-08$ & 20.22 & $11,927.8$ & 4 \\
\hline VASP_HUMAN Vasodilator-stimulated phosphoprotein & $1.37 \mathrm{E}-07$ & 30.25 & $39,805.1$ & 5 \\
\hline PSA6_RAT Proteasome subunit alpha type-6 & $2.97 \mathrm{E}-07$ & 10.17 & $27,381.8$ & 1 \\
\hline ENO1B_HUMAN Alpha-enolase, lung specific & $6.65 \mathrm{E}-07$ & 10.26 & $49,446.4$ & 1 \\
\hline HPTR_HUMAN Haptoglobin-related protein & $6.93 \mathrm{E}-07$ & 10.20 & $38,982.7$ & 1 \\
\hline
\end{tabular}


Table 1 (continued)

\begin{tabular}{|c|c|c|c|c|}
\hline & $\mathrm{P}$ (pro) & Score & MW & Hits \\
\hline IGHG3_HUMAN Ig gamma-3 chain C region & $1.04 \mathrm{E}-06$ & 20.18 & $41,260.4$ & 2 \\
\hline G3P_HUMAN Glyceraldehyde-3-phosphate dehydrogenase & $1.35 \mathrm{E}-06$ & 20.22 & $36,030.4$ & 3 \\
\hline KLKB1_HUMAN Plasma kallikrein & $2.39 \mathrm{E}-06$ & 10.18 & $71,322.8$ & 1 \\
\hline ANXA1_HUMAN Annexin A1 & $2.64 \mathrm{E}-06$ & 10.20 & $38,690.0$ & 1 \\
\hline ANXA3_HUMAN Annexin A3 & $3.79 \mathrm{E}-06$ & 24.18 & $36,352.7$ & 3 \\
\hline AMPL_HUMAN Cytosol aminopeptidase & $4.31 \mathrm{E}-06$ & 10.14 & $56,130.9$ & 1 \\
\hline S100P_HUMAN Protein S100-P & $4.65 \mathrm{E}-06$ & 10.18 & $10,393.2$ & 1 \\
\hline MMP8_HUMAN Neutrophil collagenase & $4.76 \mathrm{E}-06$ & 30.23 & $53,378.6$ & 3 \\
\hline APOE_HUMAN Apolipoprotein E & $5.02 \mathrm{E}-06$ & 50.21 & $36,131.8$ & 6 \\
\hline HBG1_PANTR Hemoglobin subunit gamma-1 & $8.24 \mathrm{E}-06$ & 20.16 & $16,130.3$ & 2 \\
\hline KV1V_HUMAN Ig kappa chain V-I region BAN & $9.89 \mathrm{E}-06$ & 10.22 & $11,832.8$ & 1 \\
\hline APOA4_HUMAN Apolipoprotein A-IV & $1.25 \mathrm{E}-05$ & 60.19 & $45,371.5$ & 7 \\
\hline KV3I_HUMAN Ig kappa chain V-III region VG precursor & $1.41 \mathrm{E}-05$ & 20.23 & $12,567.3$ & 3 \\
\hline HV3A_HUMAN Ig heavy chain V-III region TRO & $1.43 \mathrm{E}-05$ & 10.16 & $13,463.5$ & 1 \\
\hline THBG_HUMAN Thyroxine-binding globulin & $1.94 \mathrm{E}-05$ & 10.20 & $46,294.7$ & 1 \\
\hline K1C14_HUMAN Keratin, type I cytoskeletal 14 & $2.73 \mathrm{E}-05$ & 10.13 & $51,589.5$ & 1 \\
\hline IGLL1_HUMAN Immunoglobulin lambda-like polypeptide 1 & $3.37 \mathrm{E}-05$ & 38.21 & $22,948.6$ & 5 \\
\hline K1C10_HUMAN Keratin, type I cytoskeletal 10 & $5.01 \mathrm{E}-05$ & 30.19 & $59,474.8$ & 3 \\
\hline LV3A_HUMAN Ig lambda chain V-III region SH & $8.87 \mathrm{E}-05$ & 10.18 & $11,385.6$ & 1 \\
\hline CRP_HUMAN C-reactive protein & $2.33 \mathrm{E}-04$ & 20.18 & $25,022.7$ & 2 \\
\hline CBPN_HUMAN Carboxypeptidase N catalytic chain & $2.42 \mathrm{E}-04$ & 10.24 & $52,253.4$ & 1 \\
\hline CAPG_HUMAN Macrophage-capping protein & $2.55 \mathrm{E}-04$ & 20.16 & $38,493.6$ & 2 \\
\hline SAA4_HUMAN Serum amyloid A-4 protein & $2.71 \mathrm{E}-04$ & 20.14 & $14,797.3$ & 2 \\
\hline MMP3_HUMAN Stromelysin-1 & $4.23 \mathrm{E}-04$ & 10.13 & $53,943.4$ & 1 \\
\hline GDIS_HUMAN Rho GDP-dissociation inhibitor 2 & $6.94 \mathrm{E}-04$ & 20.25 & $22,973.6$ & 2 \\
\hline HV3V_HUMAN Ig heavy chain V-III region GAR & $8.20 \mathrm{E}-04$ & 10.13 & $13,079.2$ & 1 \\
\hline \multicolumn{5}{|l|}{ Fraction 34 proteins } \\
\hline CO3_HUMAN Complement C3 & $1.00 \mathrm{E}-30$ & 576.33 & $187,029.3$ & 99 \\
\hline HBA_HUMAN Hemoglobin subunit alpha & $1.00 \mathrm{E}-30$ & 230.37 & $15,247.9$ & 91 \\
\hline TTHY_HUMAN Transthyretin & $1.00 \mathrm{E}-30$ & 150.33 & $15,877.1$ & 28 \\
\hline CFAB_HUMAN Complement factor B & $3.33 \mathrm{E}-15$ & 120.35 & $85,478.6$ & 20 \\
\hline PGRP2_HUMAN N-acetylmuramoyl-L-alanine amidase & $8.88 \mathrm{E}-15$ & 290.34 & $62,178.0$ & 52 \\
\hline IGKC_HUMAN Ig kappa chain C region & $1.11 \mathrm{E}-14$ & 70.29 & $11,601.7$ & 40 \\
\hline APOA1_HUMAN Apolipoprotein A-I & $1.89 \mathrm{E}-14$ & 862.32 & $30,758.9$ & 660 \\
\hline ENOB_HUMAN Beta-enolase & $2.34 \mathrm{E}-13$ & 10.24 & $46,957.4$ & 1 \\
\hline ENOA_HUMAN Alpha-enolase & $4.31 \mathrm{E}-13$ & 40.24 & $47,139.4$ & 4 \\
\hline RETBP_PANTR Plasma retinol-binding protein & $7.37 \mathrm{E}-13$ & 58.31 & $22,995.3$ & 20 \\
\hline KV4C_HUMAN Ig kappa chain V-IV region B17 & $8.39 \mathrm{E}-13$ & 20.26 & $14,956.5$ & 3 \\
\hline IGHA1_HUMAN Ig alpha-1 chain C region & $9.03 \mathrm{E}-13$ & 110.27 & $37,630.7$ & 28 \\
\hline QSOX1_HUMAN Sulfhydryl oxidase 1 & $1.02 \mathrm{E}-12$ & 60.29 & $82,525.7$ & 6 \\
\hline HV3R_HUMAN Ig heavy chain V-III region TUR & $1.09 \mathrm{E}-12$ & 10.28 & $12,423.3$ & 2 \\
\hline MMP8_HUMAN Neutrophil collagenase & $1.19 \mathrm{E}-12$ & 60.26 & $53,378.6$ & 9 \\
\hline CO4A_HUMAN Complement C4-A & $4.03 \mathrm{E}-12$ & 190.32 & $192,649.5$ & 21 \\
\hline ITIH1_HUMAN Inter-alpha-trypsin inhibitor heavy chain H1 & $4.04 \mathrm{E}-12$ & 110.34 & $101,325.8$ & 16 \\
\hline APOL1_HUMAN Apolipoprotein-L1 & $4.42 \mathrm{E}-12$ & 10.28 & $43,947.0$ & 1 \\
\hline CO6A1_HUMAN Collagen alpha-1(VI) chain & $8.68 \mathrm{E}-12$ & 40.32 & $108,462.0$ & 6 \\
\hline LAC_HUMAN Ig lambda chain C regions & $1.47 \mathrm{E}-11$ & 50.28 & $11,229.5$ & 20 \\
\hline CFAI_HUMAN Complement factor I & $1.60 \mathrm{E}-11$ & 66.24 & $65,676.7$ & 10 \\
\hline ALBU_HUMAN Serum albumin & $2.54 \mathrm{E}-11$ & 150.26 & $69,321.6$ & 27 \\
\hline
\end{tabular}


Table 1 (continued)

\begin{tabular}{|c|c|c|c|c|}
\hline & $\mathrm{P}$ (pro) & Score & MW & Hits \\
\hline CBPB2_HUMAN Carboxypeptidase B2 & $3.13 \mathrm{E}-11$ & 10.23 & $48,381.5$ & 1 \\
\hline APOA2_HUMAN Apolipoprotein A-II & $4.64 \mathrm{E}-11$ & 118.31 & $11,167.9$ & 24 \\
\hline HEMO_HUMAN Hemopexin & $9.46 \mathrm{E}-11$ & 100.31 & $51,643.3$ & 16 \\
\hline S10A9_HUMAN Protein S100-A9 & $2.64 \mathrm{E}-10$ & 136.27 & $13,233.5$ & 33 \\
\hline HV3P_HUMAN Ig heavy chain V-III region TEI & $3.69 \mathrm{E}-10$ & 10.26 & $12,794.4$ & 1 \\
\hline ITIH2_HUMAN Inter-alpha-trypsin inhibitor heavy chain H2 & $3.86 \mathrm{E}-10$ & 30.29 & $106,369.8$ & 3 \\
\hline GSTP1_HUMAN Glutathione $S$-transferase P & $4.12 \mathrm{E}-10$ & 30.26 & $23,341.0$ & 3 \\
\hline PERM_HUMAN Myeloperoxidase & $4.13 \mathrm{E}-10$ & 90.26 & $83,815.0$ & 10 \\
\hline FIBB_HUMAN Fibrinogen beta chain & $4.90 \mathrm{E}-10$ & 60.27 & $55,892.2$ & 7 \\
\hline HBG1_PANTR Hemoglobin subunit gamma-1 & $5.83 \mathrm{E}-10$ & 30.22 & $16,130.3$ & 4 \\
\hline ANT3_HUMAN Antithrombin-III & $7.22 \mathrm{E}-10$ & 50.30 & $52,569.0$ & 8 \\
\hline HBD_HUMAN Hemoglobin subunit delta & $8.76 \mathrm{E}-10$ & 108.31 & $16,045.3$ & 20 \\
\hline ILEU_HUMAN Leukocyte elastase inhibitor & $9.91 \mathrm{E}-10$ & 10.24 & $42,714.8$ & 1 \\
\hline KV1V_HUMAN Ig kappa chain V-I region BAN & $1.08 \mathrm{E}-09$ & 10.21 & $11,832.8$ & 1 \\
\hline MUCB_HUMAN Ig mu heavy chain disease protein & $1.48 \mathrm{E}-09$ & 78.23 & $43,030.3$ & 14 \\
\hline IGHA2_HUMAN Ig alpha-2 chain C region & $1.79 \mathrm{E}-09$ & 98.22 & $36,485.1$ & 28 \\
\hline APOC2_HUMAN Apolipoprotein C-II & $2.18 \mathrm{E}-09$ & 30.26 & $11,276.8$ & 3 \\
\hline IGHM_HUMAN Ig mu chain $\mathrm{C}$ region & $2.74 \mathrm{E}-09$ & 40.24 & $49,275.6$ & 9 \\
\hline ANXA1_HUMAN Annexin A1 & $3.24 \mathrm{E}-09$ & 80.27 & $38,690.0$ & 13 \\
\hline CLUS_HUMAN Clusterin & $4.75 \mathrm{E}-09$ & 10.22 & $52,461.1$ & 2 \\
\hline KV3H_HUMAN Ig kappa chain V-III region CLL & $5.07 \mathrm{E}-09$ & 10.25 & $14,266.2$ & 1 \\
\hline HBB_HUMAN Hemoglobin subunit beta & $6.68 \mathrm{E}-09$ & 80.27 & $15,988.3$ & 18 \\
\hline IGHD_HUMAN Ig delta chain C region & $7.74 \mathrm{E}-09$ & 20.19 & $42,227.3$ & 2 \\
\hline FIBA_HUMAN Fibrinogen alpha chain & $9.00 \mathrm{E}-09$ & 20.27 & $94,914.3$ & 2 \\
\hline ANXA3_HUMAN Annexin A3 & $9.98 \mathrm{E}-09$ & 48.24 & $36,352.7$ & 5 \\
\hline IGHG3_HUMAN Ig gamma-3 chain C region & $1.14 \mathrm{E}-08$ & 10.19 & $41,260.4$ & 1 \\
\hline A2AP_HUMAN Alpha-2-antiplasmin & $1.51 \mathrm{E}-08$ & 20.24 & $54,531.2$ & 3 \\
\hline APOM_HUMAN Apolipoprotein M & $1.72 \mathrm{E}-08$ & 30.25 & $21,239.4$ & 3 \\
\hline CO6A3_HUMAN Collagen alpha-3(VI) chain & $1.86 \mathrm{E}-08$ & 34.23 & $343,337.4$ & 4 \\
\hline K2C1_HUMAN Keratin, type II cytoskeletal 1 & $3.05 \mathrm{E}-08$ & 20.35 & $65,977.9$ & 3 \\
\hline FIBG_HUMAN Fibrinogen gamma chain & $3.23 \mathrm{E}-08$ & 20.22 & $51,478.9$ & 2 \\
\hline LV4C_HUMAN Ig lambda chain V-IV region Hil & $3.65 \mathrm{E}-08$ & 10.26 & $11,509.6$ & 3 \\
\hline CO8G_HUMAN Complement component C8 gamma chain & $3.98 \mathrm{E}-08$ & 30.22 & $22,263.6$ & 4 \\
\hline APOE_HUMAN Apolipoprotein E & $4.02 \mathrm{E}-08$ & 60.27 & $36,131.8$ & 8 \\
\hline APOA4_HUMAN Apolipoprotein A-IV & $4.26 \mathrm{E}-08$ & 146.23 & $45,371.5$ & 22 \\
\hline A2MG_HUMAN Alpha-2-macroglobulin & $4.73 \mathrm{E}-08$ & 90.22 & $163,174.3$ & 9 \\
\hline LV3B_HUMAN Ig lambda chain V-III region LOI & $6.46 \mathrm{E}-08$ & 20.24 & $11,927.8$ & 2 \\
\hline KV3G_HUMAN Ig kappa chain V-III region GOL & $8.44 \mathrm{E}-08$ & 10.24 & $11,823.0$ & 1 \\
\hline KV1S_HUMAN Ig kappa chain V-I region Wes & $1.74 \mathrm{E}-07$ & 10.28 & $11,600.7$ & 1 \\
\hline HPTR_HUMAN Haptoglobin-related protein & $2.32 \mathrm{E}-07$ & 10.20 & $38,982.7$ & 1 \\
\hline PRDX2_HUMAN Peroxiredoxin-2 & $4.68 \mathrm{E}-07$ & 40.22 & $21,878.2$ & 5 \\
\hline K1C10_HUMAN Keratin, type I cytoskeletal 10 & $7.41 \mathrm{E}-07$ & 20.19 & $59,474.8$ & 2 \\
\hline ITIH4_HUMAN Inter-alpha-trypsin inhibitor heavy chain H4 & $7.44 \mathrm{E}-07$ & 100.25 & $103,261.2$ & 11 \\
\hline A1AT_HUMAN Alpha-1-antitrypsin & $9.34 \mathrm{E}-07$ & 10.18 & $46,707.1$ & 1 \\
\hline HMOX1_HUMAN Heme oxygenase 1 & $1.60 \mathrm{E}-06$ & 10.20 & $32,798.0$ & 1 \\
\hline IGJ_HUMAN Immunoglobulin J chain & $1.88 \mathrm{E}-06$ & 10.15 & $15,584.6$ & 2 \\
\hline ACTB_HUMAN Actin, cytoplasmic 1 & $3.59 \mathrm{E}-06$ & 30.19 & $41,709.7$ & 3 \\
\hline CFAH_HUMAN Complement factor $\mathrm{H}$ & $5.24 \mathrm{E}-06$ & 10.16 & $139,004.4$ & 1 \\
\hline AFAM_HUMAN Afamin & $5.26 \mathrm{E}-06$ & 10.19 & $69,024.1$ & 1 \\
\hline
\end{tabular}

\section{藻 Humana Press}


Table 1 (continued)

\begin{tabular}{|c|c|c|c|c|}
\hline & $\mathrm{P}$ (pro) & Score & MW & Hits \\
\hline TRFE_HUMAN Serotransferrin & $5.69 \mathrm{E}-06$ & 10.19 & $76,999.7$ & 1 \\
\hline A2GL_HUMAN Leucine-rich alpha-2-glycoprotein & $6.14 \mathrm{E}-06$ & 20.21 & $38,154.1$ & 2 \\
\hline CO9_HUMAN Complement component C9 & $7.89 \mathrm{E}-06$ & 20.21 & $63,132.8$ & 2 \\
\hline KV3I_HUMAN Ig kappa chain V-III region VG & $9.13 \mathrm{E}-06$ & 20.24 & $12,567.3$ & 2 \\
\hline KV2F_HUMAN Ig kappa chain V-II region RPMI 6410 & $1.13 \mathrm{E}-05$ & 10.13 & $14,697.4$ & 1 \\
\hline MMP3_HUMAN Stromelysin-1 & $2.47 \mathrm{E}-05$ & 10.17 & $53,943.4$ & 1 \\
\hline HPT_HUMAN Haptoglobin & $2.94 \mathrm{E}-05$ & 74.19 & $45,176.6$ & 13 \\
\hline S10A8_HUMAN Protein S100-A8 & $2.99 \mathrm{E}-05$ & 10.19 & $10,827.7$ & 3 \\
\hline HV1B_HUMAN Ig heavy chain V-I region HG3 & $5.54 \mathrm{E}-05$ & 10.16 & $12,937.3$ & 1 \\
\hline S11Y_HUMAN Putative S100 calcium-binding protein H_NH0456N16.1 & $6.65 \mathrm{E}-05$ & 10.20 & $11,501.9$ & 1 \\
\hline GGH_HUMAN Gamma-glutamyl hydrolase & $7.09 \mathrm{E}-05$ & 40.19 & $35,941.2$ & 4 \\
\hline SAMP_HUMAN Serum amyloid P-component & $2.25 \mathrm{E}-04$ & 30.14 & $25,371.1$ & 3 \\
\hline PROF1_HUMAN Profilin-1 & $3.64 \mathrm{E}-04$ & 10.23 & $15,044.6$ & 1 \\
\hline KV1F_HUMAN Ig kappa chain V-I region EU & $5.68 \mathrm{E}-04$ & 10.16 & $11,780.8$ & 1 \\
\hline S100P_HUMAN Protein S100-P & $6.18 \mathrm{E}-04$ & 10.18 & $10,393.2$ & 1 \\
\hline TRIM7_HUMAN Tripartite motif-containing protein 7 & $8.38 \mathrm{E}-04$ & 10.16 & $56,595.0$ & 2 \\
\hline \multicolumn{5}{|l|}{ Fraction 35 proteins } \\
\hline A2AP_HUMAN Alpha-2-antiplasmin & $1.00 \mathrm{E}-30$ & 50.30 & $54,531.2$ & 9 \\
\hline HBA_HUMAN Hemoglobin subunit alpha & $5.55 \mathrm{E}-15$ & 50.32 & $15,247.9$ & 8 \\
\hline APOA1_HUMAN Apolipoprotein A-I & $5.83 \mathrm{E}-15$ & $1,010.32$ & $30,758.9$ & 1,258 \\
\hline CO3_HUMAN Complement C3 & $1.44 \mathrm{E}-14$ & 190.35 & $187,029.3$ & 28 \\
\hline A2MG_HUMAN Alpha-2-macroglobulin & $1.44 \mathrm{E}-14$ & 60.37 & $163,174.3$ & 6 \\
\hline ACTB_HUMAN Actin, cytoplasmic 1 & $2.23 \mathrm{E}-13$ & 20.34 & $41,709.7$ & 2 \\
\hline IGKC_HUMAN Ig kappa chain C region & $2.95 \mathrm{E}-13$ & 30.25 & $11,601.7$ & 22 \\
\hline S10A6_HUMAN Protein S100-A6 & $3.49 \mathrm{E}-13$ & 30.26 & $10,173.3$ & 4 \\
\hline K2C1_HUMAN Keratin, type II cytoskeletal 1 & $1.93 \mathrm{E}-12$ & 20.25 & $65,977.9$ & 2 \\
\hline FRIL_HUMAN Ferritin light chain & $3.90 \mathrm{E}-12$ & 14.27 & $20,007.1$ & 3 \\
\hline APOA2_HUMAN Apolipoprotein A-II & $1.08 \mathrm{E}-11$ & 110.31 & $11,167.9$ & 17 \\
\hline TTHY_HUMAN Transthyretin & $1.16 \mathrm{E}-11$ & 70.30 & $15,877.1$ & 14 \\
\hline LAC_HUMAN Ig lambda chain C regions & $4.68 \mathrm{E}-11$ & 20.30 & $11,229.5$ & 13 \\
\hline PGRP2_HUMAN N-acetylmuramoyl-L-alanine amidase & $5.23 \mathrm{E}-11$ & 30.29 & $62,178.0$ & 3 \\
\hline HV3P_HUMAN Ig heavy chain V-III region TEI & $9.68 \mathrm{E}-11$ & 10.26 & $12,794.4$ & 1 \\
\hline HBD_HUMAN Hemoglobin subunit delta & $3.71 \mathrm{E}-10$ & 28.26 & $16,045.3$ & 3 \\
\hline CO4A_HUMAN Complement C4-A & $6.90 \mathrm{E}-10$ & 40.25 & $192,649.5$ & 4 \\
\hline IGHA2_HUMAN Ig alpha-2 chain C region & $1.08 \mathrm{E}-09$ & 58.22 & $36,485.1$ & 13 \\
\hline MUCB_HUMAN Ig mu heavy chain disease protein & $1.21 \mathrm{E}-09$ & 48.26 & $43,030.3$ & 6 \\
\hline ILEU_HUMAN Leukocyte elastase inhibitor & $1.50 \mathrm{E}-09$ & 30.23 & $42,714.8$ & 3 \\
\hline KV1D_HUMAN Ig kappa chain V-I region CAR & $2.61 \mathrm{E}-09$ & 10.27 & $11,696.3$ & 3 \\
\hline KAIN_HUMAN Kallistatin & $3.73 \mathrm{E}-09$ & 50.22 & $48,511.2$ & 6 \\
\hline HEMO_HUMAN Hemopexin & $4.23 \mathrm{E}-09$ & 30.23 & $51,643.3$ & 3 \\
\hline S10A8_HUMAN Protein S100-A8 & $5.29 \mathrm{E}-09$ & 10.24 & $10,827.7$ & 1 \\
\hline ALBU_HUMAN Serum albumin & $7.79 \mathrm{E}-09$ & 40.24 & $69,321.6$ & 5 \\
\hline HBB_HUMAN Hemoglobin subunit beta & $1.31 \mathrm{E}-08$ & 30.26 & $15,988.3$ & 4 \\
\hline APOA4_HUMAN Apolipoprotein A-IV & $2.29 \mathrm{E}-08$ & 40.23 & $45,371.5$ & 9 \\
\hline IGHA1_HUMAN Ig alpha-1 chain $\mathrm{C}$ region & $2.35 \mathrm{E}-08$ & 40.27 & $37,630.7$ & 5 \\
\hline CFAI_HUMAN Complement factor I & $2.62 \mathrm{E}-08$ & 20.23 & $65,676.7$ & 2 \\
\hline ITIH1_HUMAN Inter-alpha-trypsin inhibitor heavy chain H1 & $5.56 \mathrm{E}-08$ & 20.23 & $101,325.8$ & 2 \\
\hline KV3H_HUMAN Ig kappa chain V-III region CLL & $7.79 \mathrm{E}-08$ & 10.25 & $14,266.2$ & 1 \\
\hline APOE_HUMAN Apolipoprotein E & $1.90 \mathrm{E}-07$ & 20.27 & $36,131.8$ & 3 \\
\hline
\end{tabular}


Table 1 (continued)

\begin{tabular}{|c|c|c|c|c|}
\hline & $\mathrm{P}$ (pro) & Score & MW & Hits \\
\hline ITIH4_HUMAN Inter-alpha-trypsin inhibitor heavy chain H4 & $2.81 \mathrm{E}-07$ & 30.21 & $103,261.2$ & 3 \\
\hline CFAB_HUMAN Complement factor B & $3.03 \mathrm{E}-07$ & 30.24 & $85,478.6$ & 4 \\
\hline IGHM_HUMAN Ig mu chain $\mathrm{C}$ region & $3.85 \mathrm{E}-07$ & 30.23 & $49,275.6$ & 5 \\
\hline LV3B_HUMAN Ig lambda chain V-III region LOI & $1.97 \mathrm{E}-06$ & 10.25 & $11,927.8$ & 2 \\
\hline CO6A3_HUMAN Collagen alpha-3(VI) chain & $2.12 \mathrm{E}-06$ & 44.24 & $343,337.4$ & 6 \\
\hline HV3R_HUMAN Ig heavy chain V-III region TUR & $2.31 \mathrm{E}-06$ & 10.20 & $12,423.3$ & 1 \\
\hline HPT_HUMAN Haptoglobin & $4.31 \mathrm{E}-06$ & 20.18 & $45,176.6$ & 3 \\
\hline FIBA_HUMAN Fibrinogen alpha chain & $1.10 \mathrm{E}-05$ & 10.21 & $94,914.3$ & 1 \\
\hline LV4C_HUMAN Ig lambda chain V-IV region Hil & $2.21 \mathrm{E}-05$ & 10.22 & $11,509.6$ & 1 \\
\hline APOM_HUMAN Apolipoprotein M & $3.10 \mathrm{E}-05$ & 10.24 & $21,239.4$ & 1 \\
\hline PLMN_HUMAN Plasminogen & $4.83 \mathrm{E}-05$ & 10.22 & $90,510.2$ & 1 \\
\hline RETBP_PANTR Plasma retinol-binding protein & $5.79 \mathrm{E}-05$ & 20.15 & $22,995.3$ & 3 \\
\hline \multicolumn{5}{|l|}{ Fraction 36 proteins } \\
\hline CO3_HUMAN Complement C3 & $1.00 \mathrm{E}-30$ & 220.30 & $187,029.3$ & 29 \\
\hline IGHA2_HUMAN Ig alpha-2 chain C region & $1.00 \mathrm{E}-30$ & 70.34 & $36,485.1$ & 20 \\
\hline CO4B_HUMAN Complement C4-B & $2.22 \mathrm{E}-15$ & 10.33 & $192,671.6$ & 1 \\
\hline HBA_HUMAN Hemoglobin subunit alpha & $5.55 \mathrm{E}-15$ & 50.33 & $15,247.9$ & 8 \\
\hline ACTB_HUMAN Actin, cytoplasmic 1 & $7.77 \mathrm{E}-15$ & 30.32 & $41,709.7$ & 4 \\
\hline A2MG_HUMAN Alpha-2-macroglobulin & $2.22 \mathrm{E}-14$ & 100.32 & $163,174.3$ & 17 \\
\hline APOA1_HUMAN Apolipoprotein A-I & $6.08 \mathrm{E}-14$ & $1,012.33$ & $30,758.9$ & 1,155 \\
\hline A2AP_HUMAN Alpha-2-antiplasmin & $1.15 \mathrm{E}-13$ & 136.34 & $54,531.2$ & 20 \\
\hline HEMO_HUMAN Hemopexin & $1.15 \mathrm{E}-13$ & 50.21 & $51,643.3$ & 5 \\
\hline ITIH1_HUMAN Inter-alpha-trypsin inhibitor heavy chain H1 & $1.77 \mathrm{E}-13$ & 10.24 & $101,325.8$ & 1 \\
\hline K1C9_HUMAN Keratin, type I cytoskeletal 9 & $3.15 \mathrm{E}-13$ & 10.38 & $62,091.8$ & 1 \\
\hline KAIN_HUMAN Kallistatin & $6.87 \mathrm{E}-13$ & 140.28 & $48,511.2$ & 26 \\
\hline PLMN_HUMAN Plasminogen & $1.32 \mathrm{E}-12$ & 30.32 & $90,510.2$ & 3 \\
\hline APOA2_HUMAN Apolipoprotein A-II & $3.83 \mathrm{E}-12$ & 248.32 & $11,167.9$ & 167 \\
\hline KV4C_HUMAN Ig kappa chain V-IV region B17 & $3.96 \mathrm{E}-12$ & 10.28 & $14,956.5$ & 1 \\
\hline IGKC_HUMAN Ig kappa chain C region & $3.97 \mathrm{E}-12$ & 40.26 & $11,601.7$ & 23 \\
\hline RETBP_PANTR Plasma retinol-binding protein & $5.60 \mathrm{E}-12$ & 40.31 & $22,995.3$ & 7 \\
\hline K2C1_HUMAN Keratin, type II cytoskeletal 1 & $1.01 \mathrm{E}-11$ & 30.37 & $65,977.9$ & 4 \\
\hline APOA4_HUMAN Apolipoprotein A-IV & $1.79 \mathrm{E}-11$ & 70.28 & $45,371.5$ & 14 \\
\hline LAC_HUMAN Ig lambda chain $\mathrm{C}$ regions & $6.60 \mathrm{E}-11$ & 30.30 & $11,229.5$ & 13 \\
\hline KV1D_HUMAN Ig kappa chain V-I region CAR & $6.89 \mathrm{E}-11$ & 10.29 & $11,696.3$ & 1 \\
\hline HV3P_HUMAN Ig heavy chain V-III region TEI & $7.58 \mathrm{E}-11$ & 10.30 & $12,794.4$ & 1 \\
\hline HBB_HUMAN Hemoglobin subunit beta & $3.96 \mathrm{E}-10$ & 70.27 & $15,988.3$ & 13 \\
\hline FRIL_HUMAN Ferritin light chain & $4.07 \mathrm{E}-10$ & 36.27 & $20,007.1$ & 7 \\
\hline TTHY_HUMAN Transthyretin & $4.79 \mathrm{E}-10$ & 50.31 & $15,877.1$ & 6 \\
\hline CFAB_HUMAN Complement factor B & $7.78 \mathrm{E}-10$ & 20.25 & $85,478.6$ & 2 \\
\hline AMBP_HUMAN AMBP protein & $1.37 \mathrm{E}-09$ & 10.25 & $38,974.0$ & 1 \\
\hline ALBU_HUMAN Serum albumin & $1.37 \mathrm{E}-09$ & 70.24 & $69,321.6$ & 9 \\
\hline MUCB_HUMAN Ig mu heavy chain disease protein & $3.44 \mathrm{E}-09$ & 38.22 & $43,030.3$ & 6 \\
\hline KV3H_HUMAN Ig kappa chain V-III region CLL & $4.18 \mathrm{E}-09$ & 10.24 & $14,266.2$ & 1 \\
\hline A1AT_HUMAN Alpha-1-antitrypsin & $4.96 \mathrm{E}-09$ & 20.23 & $46,707.1$ & 2 \\
\hline APOL1_HUMAN Apolipoprotein-L1 & $5.95 \mathrm{E}-09$ & 30.29 & $43,947.0$ & 4 \\
\hline CO6A3_HUMAN Collagen alpha-3(VI) chain & $7.12 \mathrm{E}-09$ & 48.23 & $343,337.4$ & 6 \\
\hline S10A9_HUMAN Protein S100-A9 & $8.28 \mathrm{E}-09$ & 10.26 & $13,233.5$ & 1 \\
\hline CFAI_HUMAN Complement factor I & $1.04 \mathrm{E}-08$ & 46.24 & $65,676.7$ & 5 \\
\hline IGHG3_HUMAN Ig gamma-3 chain C region & $1.24 \mathrm{E}-08$ & 10.17 & $41,260.4$ & 1 \\
\hline
\end{tabular}


Table 1 (continued)

\begin{tabular}{|c|c|c|c|c|}
\hline & $\mathrm{P}$ (pro) & Score & MW & Hits \\
\hline FIBA_HUMAN Fibrinogen alpha chain & $1.61 \mathrm{E}-08$ & 20.22 & $94,914.3$ & 2 \\
\hline APOE_HUMAN Apolipoprotein E & $3.40 \mathrm{E}-08$ & 20.27 & $36,131.8$ & 3 \\
\hline IGHA1_HUMAN Ig alpha-1 chain C region & $6.15 \mathrm{E}-08$ & 30.24 & $37,630.7$ & 5 \\
\hline IGHM_HUMAN Ig mu chain C region & $1.50 \mathrm{E}-07$ & 40.24 & $49,275.6$ & 8 \\
\hline ACTS_HUMAN Actin, alpha skeletal muscle & $2.46 \mathrm{E}-07$ & 50.23 & $42,023.9$ & 6 \\
\hline TALDO_HUMAN Transaldolase & $2.88 \mathrm{E}-07$ & 20.19 & $37,516.5$ & 2 \\
\hline ANT3_HUMAN Antithrombin-III & $1.16 \mathrm{E}-06$ & 10.22 & $52,569.0$ & 1 \\
\hline CO4A_HUMAN Complement C4-A & $6.92 \mathrm{E}-06$ & 26.18 & $192,649.5$ & 3 \\
\hline CO8G_HUMAN Complement component C8 gamma chain & $1.20 \mathrm{E}-05$ & 10.20 & $22,263.6$ & 1 \\
\hline HPT_HUMAN Haptoglobin & $1.99 \mathrm{E}-05$ & 28.19 & $45,176.6$ & 4 \\
\hline ILEU_HUMAN Leukocyte elastase inhibitor & $2.13 \mathrm{E}-05$ & 20.23 & $42,714.8$ & 2 \\
\hline IGJ_HUMAN Immunoglobulin J chain & $2.93 \mathrm{E}-05$ & 10.16 & $15,584.6$ & 1 \\
\hline LV3B_HUMAN Ig lambda chain V-III region LOI & $3.63 \mathrm{E}-05$ & 10.20 & $11,927.8$ & 1 \\
\hline M6PBP_HUMAN Mannose-6-phosphate receptor-binding protein 1 & $3.95 \mathrm{E}-04$ & 10.22 & $47,018.0$ & 1 \\
\hline PROF1_HUMAN Profilin-1 & $7.14 \mathrm{E}-04$ & 10.20 & $15,044.6$ & 1 \\
\hline \multicolumn{5}{|l|}{ Fraction 37 proteins } \\
\hline IGHA1_HUMAN Ig alpha-1 chain C region & $1.00 \mathrm{E}-30$ & 70.29 & $37,630.7$ & 17 \\
\hline K2C1_HUMAN Keratin, type II cytoskeletal 1 & $1.11 \mathrm{E}-16$ & 60.39 & $65,977.9$ & 7 \\
\hline ACTB_HUMAN Actin, cytoplasmic 1 & $5.55 \mathrm{E}-16$ & 150.32 & $41,709.7$ & 19 \\
\hline K1C9_HUMAN Keratin, type I cytoskeletal 9 & $1.11 \mathrm{E}-15$ & 44.38 & $62,091.8$ & 6 \\
\hline APOB_HUMAN Apolipoprotein B-100 & $1.33 \mathrm{E}-15$ & 398.38 & $515,241.6$ & 53 \\
\hline A2AP_HUMAN Alpha-2-antiplasmin & $3.33 \mathrm{E}-15$ & 222.33 & $54,531.2$ & 32 \\
\hline PLMN_HUMAN Plasminogen & $4.44 \mathrm{E}-15$ & 50.33 & $90,510.2$ & 8 \\
\hline CO3_HUMAN Complement C3 & $9.88 \mathrm{E}-15$ & 352.35 & $187,029.3$ & 49 \\
\hline APOE_HUMAN Apolipoprotein E & $7.77 \mathrm{E}-14$ & 50.29 & $36,131.8$ & 5 \\
\hline IGKC_HUMAN Ig kappa chain C region & $1.58 \mathrm{E}-13$ & 70.30 & $11,601.7$ & 30 \\
\hline LKHA4_HUMAN Leukotriene A-4 hydrolase & $1.75 \mathrm{E}-13$ & 50.26 & $69,241.3$ & 5 \\
\hline PGRP2_HUMAN $N$-acetylmuramoyl-L-alanine amidase & $3.96 \mathrm{E}-13$ & 20.27 & $62,178.0$ & 2 \\
\hline APOL1_HUMAN Apolipoprotein-L1 & $4.22 \mathrm{E}-13$ & 150.31 & $43,947.0$ & 23 \\
\hline A2MG_HUMAN Alpha-2-macroglobulin & $5.13 \mathrm{E}-13$ & 380.37 & $163,174.3$ & 58 \\
\hline ANGT_HUMAN Angiotensinogen & $2.56 \mathrm{E}-12$ & 50.31 & $53,120.6$ & 5 \\
\hline CO4A_HUMAN Complement C4-A & $5.35 \mathrm{E}-12$ & 36.27 & $192,649.5$ & 4 \\
\hline KAIN_HUMAN Kallistatin & $6.59 \mathrm{E}-12$ & 40.30 & $48,511.2$ & 5 \\
\hline APOA2_HUMAN Apolipoprotein A-II & $8.45 \mathrm{E}-12$ & 174.33 & $11,167.9$ & 68 \\
\hline APOA1_HUMAN Apolipoprotein A-I & $8.67 \mathrm{E}-12$ & 912.32 & $30,758.9$ & 918 \\
\hline PLTP_HUMAN Phospholipid transfer protein & $2.02 \mathrm{E}-11$ & 30.26 & $54,704.7$ & 3 \\
\hline KV4C_HUMAN Ig kappa chain V-IV region B17 & $2.83 \mathrm{E}-11$ & 20.26 & $14,956.5$ & 3 \\
\hline S10A6_HUMAN Protein S100-A6 & $2.88 \mathrm{E}-11$ & 10.25 & $10,173.3$ & 1 \\
\hline CO5_HUMAN Complement C5 & $2.96 \mathrm{E}-11$ & 110.25 & $188,185.3$ & 11 \\
\hline RETBP_PANTR Plasma retinol-binding protein & $3.46 \mathrm{E}-11$ & 50.29 & $22,995.3$ & 12 \\
\hline HBA_HUMAN Hemoglobin subunit alpha & $5.76 \mathrm{E}-11$ & 30.20 & $15,247.9$ & 5 \\
\hline ITIH1_HUMAN Inter-alpha-trypsin inhibitor heavy chain H1 & $6.33 \mathrm{E}-11$ & 10.30 & $101,325.8$ & 1 \\
\hline LAC_HUMAN Ig lambda chain C regions & $8.48 \mathrm{E}-11$ & 50.30 & $11,229.5$ & 15 \\
\hline KV1D_HUMAN Ig kappa chain V-I region CAR & $1.34 \mathrm{E}-10$ & 20.26 & $11,696.3$ & 4 \\
\hline HEMO_HUMAN Hemopexin & $1.43 \mathrm{E}-10$ & 88.25 & $51,643.3$ & 11 \\
\hline CO6A3_HUMAN Collagen alpha-3(VI) chain & $1.90 \mathrm{E}-10$ & 38.22 & $343,337.4$ & 5 \\
\hline HV3R_HUMAN Ig heavy chain V-III region TUR & $2.35 \mathrm{E}-10$ & 10.26 & $12,423.3$ & 1 \\
\hline ITIH2_HUMAN Inter-alpha-trypsin inhibitor heavy chain H2 & $3.54 \mathrm{E}-10$ & 100.25 & $106,369.8$ & 15 \\
\hline TTHY_HUMAN Transthyretin & $3.86 \mathrm{E}-10$ & 30.34 & $15,877.1$ & 4 \\
\hline
\end{tabular}


Table 1 (continued)

\begin{tabular}{|c|c|c|c|c|}
\hline & $\mathrm{P}($ pro) & Score & MW & Hits \\
\hline HBD_HUMAN Hemoglobin subunit delta & $4.49 \mathrm{E}-10$ & 38.25 & $16,045.3$ & 6 \\
\hline AMBP_HUMAN AMBP protein & $7.76 \mathrm{E}-10$ & 10.24 & $38,974.0$ & 1 \\
\hline CO8G_HUMAN Complement component C8 gamma chain & $8.36 \mathrm{E}-10$ & 30.23 & $22,263.6$ & 4 \\
\hline IGHA2_HUMAN Ig alpha-2 chain $\mathrm{C}$ region & 4.57E-09 & 68.25 & $36,485.1$ & 13 \\
\hline ALBU_HUMAN Serum albumin & $4.66 \mathrm{E}-09$ & 60.24 & $69,321.6$ & 7 \\
\hline HBB_HUMAN Hemoglobin subunit beta & $5.88 \mathrm{E}-09$ & 40.27 & $15,988.3$ & 6 \\
\hline FIBB_HUMAN Fibrinogen beta chain & $7.26 \mathrm{E}-09$ & 10.24 & $55,892.2$ & 1 \\
\hline CFAI_HUMAN Complement factor I & $1.06 \mathrm{E}-08$ & 46.21 & $65,676.7$ & 5 \\
\hline MUCB_HUMAN Ig mu heavy chain disease protein & $1.38 \mathrm{E}-08$ & 58.24 & $43,030.3$ & 11 \\
\hline LV3B_HUMAN Ig lambda chain V-III region LOI & $1.87 \mathrm{E}-08$ & 10.23 & $11,927.8$ & 1 \\
\hline POSTN_HUMAN Periostin & $2.21 \mathrm{E}-08$ & 34.25 & $93,255.4$ & 5 \\
\hline KV3H_HUMAN Ig kappa chain V-III region CLL & $2.88 \mathrm{E}-08$ & 10.24 & $14,266.2$ & 1 \\
\hline S10A9_HUMAN Protein S100-A9 & $3.71 \mathrm{E}-08$ & 20.17 & $13,233.5$ & 2 \\
\hline IGHM_HUMAN Ig mu chain $\mathrm{C}$ region & $4.69 \mathrm{E}-08$ & 30.24 & $49,275.6$ & 7 \\
\hline KV3G_HUMAN Ig kappa chain V-III region GOL & $5.54 \mathrm{E}-08$ & 10.22 & $11,823.0$ & 1 \\
\hline FIBA_HUMAN Fibrinogen alpha chain & $7.08 \mathrm{E}-08$ & 20.26 & $94,914.3$ & 2 \\
\hline CO2_HUMAN Complement $\mathrm{C} 2$ & $7.13 \mathrm{E}-08$ & 20.22 & $83,214.4$ & 2 \\
\hline LDHB_HUMAN L-lactate dehydrogenase B chain & $8.55 \mathrm{E}-08$ & 10.18 & $36,615.2$ & 1 \\
\hline PEDF_HUMAN Pigment epithelium-derived factor & $9.52 \mathrm{E}-08$ & 30.17 & $46,313.4$ & 3 \\
\hline AACT_HUMAN Alpha-1-antichymotrypsin & $1.12 \mathrm{E}-07$ & 10.27 & $47,620.6$ & 1 \\
\hline ITIH4_HUMAN Inter-alpha-trypsin inhibitor heavy chain H4 & $1.20 \mathrm{E}-07$ & 20.22 & $103,261.2$ & 3 \\
\hline ANT3_HUMAN Antithrombin-III & $1.79 \mathrm{E}-07$ & 10.27 & $52,569.0$ & 1 \\
\hline KV1V_HUMAN Ig kappa chain V-I region BAN & $2.30 \mathrm{E}-07$ & 10.22 & $11,832.8$ & 1 \\
\hline ACTS_HUMAN Actin, alpha skeletal muscle & $2.70 \mathrm{E}-07$ & 96.22 & $42,023.9$ & 14 \\
\hline APOA4_HUMAN Apolipoprotein A-IV & $3.06 \mathrm{E}-07$ & 30.18 & $45,371.5$ & 4 \\
\hline HRG_HUMAN Histidine-rich glycoprotein & $8.44 \mathrm{E}-07$ & 10.18 & $59,540.9$ & 1 \\
\hline ADIPO_HUMAN Adiponectin & $8.48 \mathrm{E}-07$ & 10.23 & $26,397.0$ & 1 \\
\hline HV3P_HUMAN Ig heavy chain V-III region TEI & $1.03 \mathrm{E}-06$ & 10.26 & $12,794.4$ & 1 \\
\hline SAMP_HUMAN Serum amyloid P-component & $2.09 \mathrm{E}-06$ & 10.17 & $25,371.1$ & 1 \\
\hline TALDO_HUMAN Transaldolase & $2.15 \mathrm{E}-06$ & 30.20 & $37,516.5$ & 5 \\
\hline KV3I_HUMAN Ig kappa chain V-III region VG & $6.88 \mathrm{E}-06$ & 10.23 & $12,567.3$ & 2 \\
\hline VINC_HUMAN Vinculin & $8.61 \mathrm{E}-06$ & 30.23 & $123,721.9$ & 3 \\
\hline LDHA_HUMAN L-lactate dehydrogenase A chain & $1.07 \mathrm{E}-05$ & 10.15 & $36,665.4$ & 1 \\
\hline IGJ_HUMAN Immunoglobulin J chain & $1.38 \mathrm{E}-05$ & 10.15 & $15,584.6$ & 1 \\
\hline HPT_HUMAN Haptoglobin & $2.95 \mathrm{E}-05$ & 64.24 & $45,176.6$ & 7 \\
\hline FA12_HUMAN Coagulation factor XII & $3.00 \mathrm{E}-05$ & 10.23 & $67,774.1$ & 1 \\
\hline LV4C_HUMAN Ig lambda chain V-IV region Hil & $3.06 \mathrm{E}-05$ & 10.20 & $11,509.6$ & 1 \\
\hline PROF1_HUMAN Profilin-1 & $1.12 \mathrm{E}-04$ & 10.21 & $15,044.6$ & 1 \\
\hline HV3A_HUMAN Ig heavy chain V-III region TRO & $1.46 \mathrm{E}-04$ & 10.16 & $13,463.5$ & 1 \\
\hline BGH3_HUMAN Transforming growth factor-beta-induced protein ig-h3 & $1.76 \mathrm{E}-04$ & 10.18 & $74,634.1$ & 1 \\
\hline CSN8_HUMAN COP9 signalosome complex subunit 8 & $1.78 \mathrm{E}-04$ & 10.16 & $23,211.0$ & 1 \\
\hline O52A5_HUMAN Olfactory receptor 52A5 & $3.20 \mathrm{E}-04$ & 10.12 & $35,931.3$ & 1 \\
\hline \multicolumn{5}{|l|}{ Fraction 38 proteins } \\
\hline A2AP_HUMAN Alpha-2-antiplasmin & $1.11 \mathrm{E}-16$ & 108.31 & $54,531.2$ & 15 \\
\hline A2MG_HUMAN Alpha-2-macroglobulin & $2.22 \mathrm{E}-15$ & 538.38 & $163,174.3$ & 88 \\
\hline APOA1_HUMAN Apolipoprotein A-I & $2.55 \mathrm{E}-14$ & 350.33 & $30,758.9$ & 389 \\
\hline IGHA1_HUMAN Ig alpha-1 chain C region & $3.00 \mathrm{E}-14$ & 40.25 & $37,630.7$ & 16 \\
\hline PGRP2_HUMAN N-acetylmuramoyl-L-alanine amidase & $6.44 \mathrm{E}-14$ & 10.29 & $62,178.0$ & 3 \\
\hline ACTB_HUMAN Actin, cytoplasmic 1 & $1.98 \mathrm{E}-13$ & 90.32 & $41,709.7$ & 13 \\
\hline
\end{tabular}


Table 1 (continued)

\begin{tabular}{|c|c|c|c|c|}
\hline & $\mathrm{P}($ pro) & Score & MW & Hits \\
\hline K2C1_HUMAN Keratin, type II cytoskeletal 1 & $3.56 \mathrm{E}-13$ & 130.36 & $65,977.9$ & 16 \\
\hline APOL1_HUMAN Apolipoprotein-L1 & $1.43 \mathrm{E}-12$ & 80.29 & $43,947.0$ & 10 \\
\hline PLMN_HUMAN Plasminogen & $1.47 \mathrm{E}-12$ & 20.31 & $90,510.2$ & 3 \\
\hline IGKC_HUMAN Ig kappa chain C region & $5.07 \mathrm{E}-12$ & 60.29 & $11,601.7$ & 43 \\
\hline HV3R_HUMAN Ig heavy chain V-III region TUR & $5.17 \mathrm{E}-12$ & 10.29 & $12,423.3$ & 2 \\
\hline K1C10_HUMAN Keratin, type I cytoskeletal 10 & $5.49 \mathrm{E}-12$ & 80.31 & $59,474.8$ & 9 \\
\hline TLN1_HUMAN Talin-1 & $5.71 \mathrm{E}-12$ & 40.28 & $269,596.3$ & 5 \\
\hline APOB_HUMAN Apolipoprotein B-100 & $6.46 \mathrm{E}-12$ & 498.35 & $515,241.6$ & 82 \\
\hline HBA_HUMAN Hemoglobin subunit alpha & $2.90 \mathrm{E}-11$ & 50.30 & $15,247.9$ & 8 \\
\hline CO3_HUMAN Complement C3 & $2.99 \mathrm{E}-11$ & 480.30 & $187,029.3$ & 69 \\
\hline KV4C_HUMAN Ig kappa chain V-IV region B17 & $5.13 \mathrm{E}-11$ & 40.24 & $14,956.5$ & 5 \\
\hline LV4C_HUMAN Ig lambda chain V-IV region Hil & $7.27 \mathrm{E}-11$ & 10.24 & $11,509.6$ & 1 \\
\hline LAC_HUMAN Ig lambda chain $\mathrm{C}$ regions & $1.33 \mathrm{E}-10$ & 20.28 & $11,229.5$ & 17 \\
\hline APOA2_HUMAN Apolipoprotein A-II & $1.34 \mathrm{E}-10$ & 120.32 & $11,167.9$ & 20 \\
\hline TTHY_HUMAN Transthyretin & $2.49 \mathrm{E}-10$ & 60.23 & $15,877.1$ & 8 \\
\hline HEMO_HUMAN Hemopexin & $2.93 \mathrm{E}-10$ & 60.25 & $51,643.3$ & 15 \\
\hline S10A6_HUMAN Protein S100-A6 & $4.78 \mathrm{E}-10$ & 20.22 & $10,173.3$ & 2 \\
\hline ACTS_HUMAN Actin, alpha skeletal muscle & $1.07 \mathrm{E}-09$ & 28.20 & $42,023.9$ & 4 \\
\hline RETBP_PANTR Plasma retinol-binding protein & $1.47 \mathrm{E}-09$ & 100.30 & $22,995.3$ & 31 \\
\hline ENOA_HUMAN Alpha-enolase & $1.49 \mathrm{E}-09$ & 30.24 & $47,139.4$ & 3 \\
\hline ANT3_HUMAN Antithrombin-III & $2.72 \mathrm{E}-09$ & 20.25 & $52,569.0$ & 2 \\
\hline HBB_HUMAN Hemoglobin subunit beta & $3.32 \mathrm{E}-09$ & 50.25 & $15,988.3$ & 12 \\
\hline CFAB_HUMAN Complement factor B & $3.59 \mathrm{E}-09$ & 40.23 & $85,478.6$ & 4 \\
\hline S10A9_HUMAN Protein S100-A9 & $3.63 \mathrm{E}-09$ & 20.21 & $13,233.5$ & 2 \\
\hline K1C9_HUMAN Keratin, type I cytoskeletal 9 & $4.51 \mathrm{E}-09$ & 90.40 & $62,091.8$ & 13 \\
\hline CO8G_HUMAN Complement component C8 gamma chain & $5.21 \mathrm{E}-09$ & 30.21 & $22,263.6$ & 3 \\
\hline CO5_HUMAN Complement C5 & $6.03 \mathrm{E}-09$ & 160.23 & $188,185.3$ & 17 \\
\hline DCTN2_HUMAN Dynactin subunit 2 & $9.83 \mathrm{E}-09$ & 30.20 & $44,203.9$ & 3 \\
\hline CO6A3_HUMAN Collagen alpha-3(VI) chain & $1.06 \mathrm{E}-08$ & 10.19 & $343,337.4$ & 1 \\
\hline MUCB_HUMAN Ig mu heavy chain disease protein & $1.58 \mathrm{E}-08$ & 68.23 & $43,030.3$ & 23 \\
\hline CO4A_HUMAN Complement C4-A & $1.67 \mathrm{E}-08$ & 62.23 & $192,649.5$ & 7 \\
\hline IGHA2_HUMAN Ig alpha-2 chain $\mathrm{C}$ region & $1.83 \mathrm{E}-08$ & 100.23 & $36,485.1$ & 20 \\
\hline IGHM_HUMAN RecName: Full=Ig mu chain $\mathrm{C}$ region & $2.08 \mathrm{E}-08$ & 30.23 & $49,275.6$ & 9 \\
\hline HRG_HUMAN Histidine-rich glycoprotein & $2.20 \mathrm{E}-08$ & 20.23 & $59,540.9$ & 2 \\
\hline 6PGD_HUMAN 6-phosphogluconate dehydrogenase, decarboxylating & $2.72 \mathrm{E}-08$ & 10.23 & $53,106.0$ & 1 \\
\hline SAMP_HUMAN Serum amyloid P-component & $2.99 \mathrm{E}-08$ & 30.20 & $25,371.1$ & 4 \\
\hline ITIH4_HUMAN Inter-alpha-trypsin inhibitor heavy chain H4 & $5.84 \mathrm{E}-08$ & 30.22 & $103,261.2$ & 3 \\
\hline TKT_HUMAN Transketolase & $7.66 \mathrm{E}-08$ & 20.19 & $67,834.9$ & 3 \\
\hline BGH3_HUMAN Transforming growth factor-beta-induced protein ig-h3 & $1.10 \mathrm{E}-07$ & 40.20 & $74,634.1$ & 4 \\
\hline ALBU_HUMAN Serum albumin & $1.25 \mathrm{E}-07$ & 130.25 & $69,321.6$ & 18 \\
\hline SPB9_HUMAN Serpin B9 & $1.27 \mathrm{E}-07$ & 80.19 & $42,376.4$ & 8 \\
\hline HBD_HUMAN Hemoglobin subunit delta & $1.52 \mathrm{E}-07$ & 28.19 & $16,045.3$ & 3 \\
\hline APOA4_HUMAN Apolipoprotein A-IV & $2.42 \mathrm{E}-07$ & 40.20 & $45,371.5$ & 4 \\
\hline APOE_HUMAN Apolipoprotein E & $2.58 \mathrm{E}-07$ & 20.25 & $36,131.8$ & 2 \\
\hline HPT_HUMAN Haptoglobin & $2.74 \mathrm{E}-07$ & 102.23 & $45,176.6$ & 18 \\
\hline CFAI_HUMAN Complement factor I & $2.85 \mathrm{E}-07$ & 56.23 & $65,676.7$ & 8 \\
\hline ADIPO_HUMAN Adiponectin & $4.68 \mathrm{E}-07$ & 10.23 & $26,397.0$ & 1 \\
\hline ANGT_HUMAN Angiotensinogen & $4.90 \mathrm{E}-07$ & 30.22 & $53,120.6$ & 3 \\
\hline IGLL1_HUMAN Immunoglobulin lambda-like polypeptide 1 & $7.41 \mathrm{E}-07$ & 10.19 & $22,948.6$ & 1 \\
\hline
\end{tabular}


Table 1 (continued)

\begin{tabular}{|c|c|c|c|c|}
\hline & $\mathrm{P}$ (pro) & Score & MW & Hits \\
\hline ENO1B_HUMAN Alpha-enolase, lung specific & $9.50 \mathrm{E}-07$ & 20.24 & $49,446.4$ & 2 \\
\hline PEDF_HUMAN Pigment epithelium-derived factor & $9.80 \mathrm{E}-07$ & 46.18 & $46,313.4$ & 5 \\
\hline KV3G_HUMAN Ig kappa chain V-III region GOL & $1.03 \mathrm{E}-06$ & 10.19 & $11,823.0$ & 2 \\
\hline KAIN_HUMAN Kallistatin & $1.10 \mathrm{E}-06$ & 10.20 & $48,511.2$ & 1 \\
\hline ENOB_HUMAN Beta-enolase & $1.18 \mathrm{E}-06$ & 10.23 & $46,957.4$ & 2 \\
\hline S10A8_HUMAN Protein S100-A8 & $1.41 \mathrm{E}-06$ & 10.22 & $10,827.7$ & 1 \\
\hline KV1D_HUMAN Ig kappa chain V-I region CAR & $2.42 \mathrm{E}-06$ & 20.24 & $11,696.3$ & 2 \\
\hline LV3B_HUMAN Ig lambda chain V-III region LOI & $2.43 \mathrm{E}-06$ & 30.24 & $11,927.8$ & 4 \\
\hline IGJ_HUMAN Immunoglobulin J chain & $3.40 \mathrm{E}-06$ & 10.16 & $15,584.6$ & 1 \\
\hline ITIH2_HUMAN Inter-alpha-trypsin inhibitor heavy chain H2 & $4.00 \mathrm{E}-06$ & 40.20 & $106,369.8$ & 4 \\
\hline K22E_HUMAN Keratin, type II cytoskeletal 2 epidermal & $6.60 \mathrm{E}-06$ & 20.23 & $65,825.4$ & 2 \\
\hline HV3P_HUMAN Ig heavy chain V-III region TEI & $8.90 \mathrm{E}-06$ & 10.26 & $12,794.4$ & 3 \\
\hline PSME1_HUMAN Proteasome activator complex subunit 1 & $9.97 \mathrm{E}-06$ & 20.15 & $28,705.0$ & 2 \\
\hline PLTP_HUMAN Phospholipid transfer protein & $1.10 \mathrm{E}-05$ & 40.20 & $54,704.7$ & 5 \\
\hline CO2_HUMAN Complement C2 & $1.43 \mathrm{E}-05$ & 10.23 & $83,214.4$ & 1 \\
\hline KV3H_HUMAN Ig kappa chain V-III region CLL & $1.66 \mathrm{E}-05$ & 20.19 & $14,266.2$ & 2 \\
\hline TETN_HUMAN Tetranectin & $1.82 \mathrm{E}-05$ & 10.18 & $22,552.3$ & 1 \\
\hline LV3A_HUMAN Ig lambda chain V-III region SH & $1.87 \mathrm{E}-05$ & 10.21 & $11,385.6$ & 2 \\
\hline LDHA_HUMAN L-lactate dehydrogenase A chain & $1.89 \mathrm{E}-05$ & 10.19 & $36,665.4$ & 1 \\
\hline LDHB_HUMAN L-lactate dehydrogenase B chain & $2.04 \mathrm{E}-05$ & 30.22 & $36,615.2$ & 3 \\
\hline C1QB_HUMAN Complement C1q subcomponent subunit B & $3.63 \mathrm{E}-05$ & 10.23 & $26,442.4$ & 1 \\
\hline ILEU_HUMAN Leukocyte elastase inhibitor & $5.71 \mathrm{E}-05$ & 10.17 & $42,714.8$ & 1 \\
\hline IPSP_HUMAN Plasma serine protease inhibitor & $7.70 \mathrm{E}-05$ & 10.16 & $45,672.8$ & 1 \\
\hline IGHD_HUMAN Ig delta chain $\mathrm{C}$ region & $1.11 \mathrm{E}-04$ & 10.17 & $42,227.3$ & 1 \\
\hline FIBB_HUMAN Fibrinogen beta chain & $2.02 \mathrm{E}-04$ & 10.18 & $55,892.2$ & 1 \\
\hline IGHG3_HUMAN Ig gamma-3 chain C region & $2.10 \mathrm{E}-04$ & 10.16 & $41,260.4$ & 1 \\
\hline HV3V_HUMAN Ig heavy chain V-III region GAR & $2.52 \mathrm{E}-04$ & 10.17 & $13,079.2$ & 1 \\
\hline HPTR_HUMAN Haptoglobin-related protein & $2.62 \mathrm{E}-04$ & 10.20 & $38,982.7$ & 1 \\
\hline C1QC_HUMAN Complement C1q subcomponent subunit C & $2.92 \mathrm{E}-04$ & 10.19 & $25,757.1$ & 1 \\
\hline KV3I_HUMAN Ig kappa chain V-III region VG & $3.01 \mathrm{E}-04$ & 10.20 & $12,567.3$ & 1 \\
\hline PROF1_HUMAN Profilin-1 & $3.49 \mathrm{E}-04$ & 10.17 & $15,044.6$ & 1 \\
\hline K1C25_HUMAN Keratin, type I cytoskeletal 25 & $7.85 \mathrm{E}-04$ & 10.15 & $49,287.4$ & 1 \\
\hline
\end{tabular}

the protein array analysis, the mass spectrometry analysis did not yield a protein identification. Both the protein arrays and HPLC chromatographs appear to be more sensitive at detecting proteins than the mass spectrometry.

In order to obtain enough material for mass spectrometry analysis, SF from nine different patients was pooled (total volume of $50 \mathrm{ml}$ ) and used for a large-scale procedure. After protein depletion, approximately $20 \mathrm{mg}$ of depleted SF material was used to perform four first-dimension chromatofocusing runs (Fig. 3a). The fractions that eluted at $\mathrm{pH}$ 5.63-5.45 were combined to perform one second-dimension RP-HPLC run (Fig. 3b). Fractions corresponding to the region that tested positive for RA serum binding in the protein array were digested with trypsin and analyzed by $\mathrm{LC} /$ MS/MS. SEQUEST searches were performed using the SwissProt database limiting the search to tryptic peptides.
Table 1 lists the proteins found in these fractions and the corresponding peptides are included as supplementary material (Supplement Table 1).

The candidate autoantigen specifically detected by the RA serum was estimated to be in fractions 20-22. Amongst other proteins, these fractions contained fibrinogen, a known autoantigen that can be citrullinated in vivo. Fibrinogen alpha (FIBA_HUMAN, SwissProt Accession \# P02671) was identified in fraction 20 with nine unique peptide hits (15\% sequence coverage) and in fraction 22 with 18 unique peptides ( $24 \%$ sequence coverage). Altogether, peptides were only found originating from the center of the FIBA protein (amino acids 250-599) corresponding to the alphaC domain of fibrin (amino acids 239-629). Figure 4 shows the sequence of the fibrin alphaC domain with the amino acids identified by mass spectrom- 
Fig. 4 The fibrin alphaC domain (239-629) with the regions identified by mass spectrometry in bold and the FIBA 259-287 underlined. Arrows point to modifications

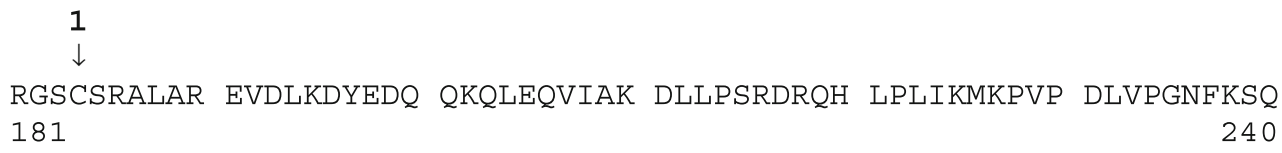

$\begin{array}{lccclr} & 2 & 3 & 4 & 5 & 6 \\ & \downarrow & \downarrow & \downarrow & \downarrow & \downarrow \\ \text { LQKVPPEWKA } & \text { LTDMPQMRME } & \text { LERPGGNEIT } & \text { RGGSTSYGTG } & \text { SETESPRNPS } & \text { SAGSWNSGSS } \\ 241 & & & & & \end{array}$

GPGSTGNRNP GSSGTGGTAT WKPGSSGPGS TGSWNSGSSG TGSTGNQNPG SPRPGSTGTW

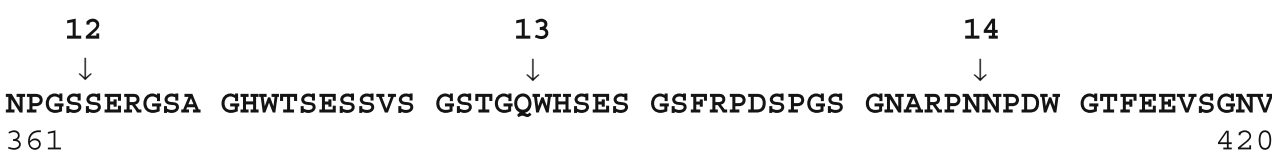

$\begin{array}{lccccc} & 15 & 16 & 17 & 18 & 19 \\ \text { SPGTRREYHT } & \text { EKLVTSKGDK } & \text { ELRTGKEKVT } & \text { SGSTTTTRRS } & \text { CSKTVTKTVI } & \text { GPDGHKEVTK } \\ 421 & & & & & \end{array}$

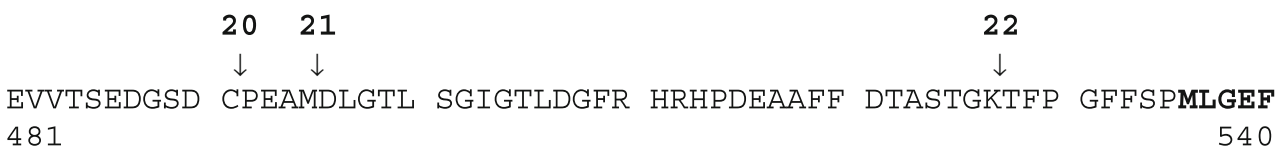

\begin{tabular}{|c|c|c|c|c|c|}
\hline 23 & 24 & & $25-29$ & 30 & 31 \\
\hline$\downarrow$ & $\downarrow$ & & $\downarrow \downarrow \downarrow \downarrow \downarrow$ & $\downarrow$ & $\downarrow$ \\
\hline SETESRGSE & SGIFTNTKES & SSHHPGIAEF & PSRGKSSSYS & KQFTSSTSYN & \\
\hline
\end{tabular}

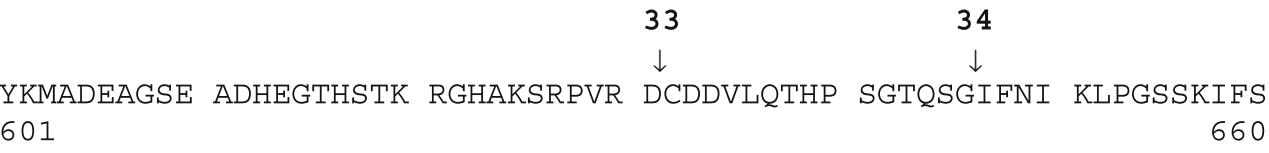

etry in bold and arrows pointing to any modifications. Peptides from the center region were not identified due a lysine-/arginine-rich area followed by two cross-linked amino acids (Ser461-Ser491).

Careful examination of the mass spectra assigned to arginine-containing FIBA peptides led to the assignment of a citrullinated peptide corresponding to FIBA 259-287. The calculated mass obtained from the mass spectrum (Fig. 5) was 1 Da higher than the calculated mass for the unmodified peptide. The lower resolution MS/MS spectrum obtained with the ion trap part of the LTQ-FT did not allow determination of the exact location of the modification. However, a number of other fragments corresponding to parts of the peptide (Table 2) were observed in the spectra obtained with the ion cyclotron resonance (ICR) analyzer of the LTQ-FT, which provided precise mass measurements. There are four sites on the FIBA 259-287 peptide that could possibly be modified resulting in a mass shift of +1 Da. The peptide contains three arginines that can be citrullinated and an asparagine that can be deaminated to form aspartic acid (Fig. 5). All fragments that did not show the expected tryptic cleavage at Arg 271 showed observed masses that were $1 \mathrm{Da}$ higher than the expected mass for the unmodified form. This is consistent with the known failure of trypsin to cleave citrullinated Arg residues. Thus, only conversion of Arg 271 to citrulline is 
Fig. 4 (continued)

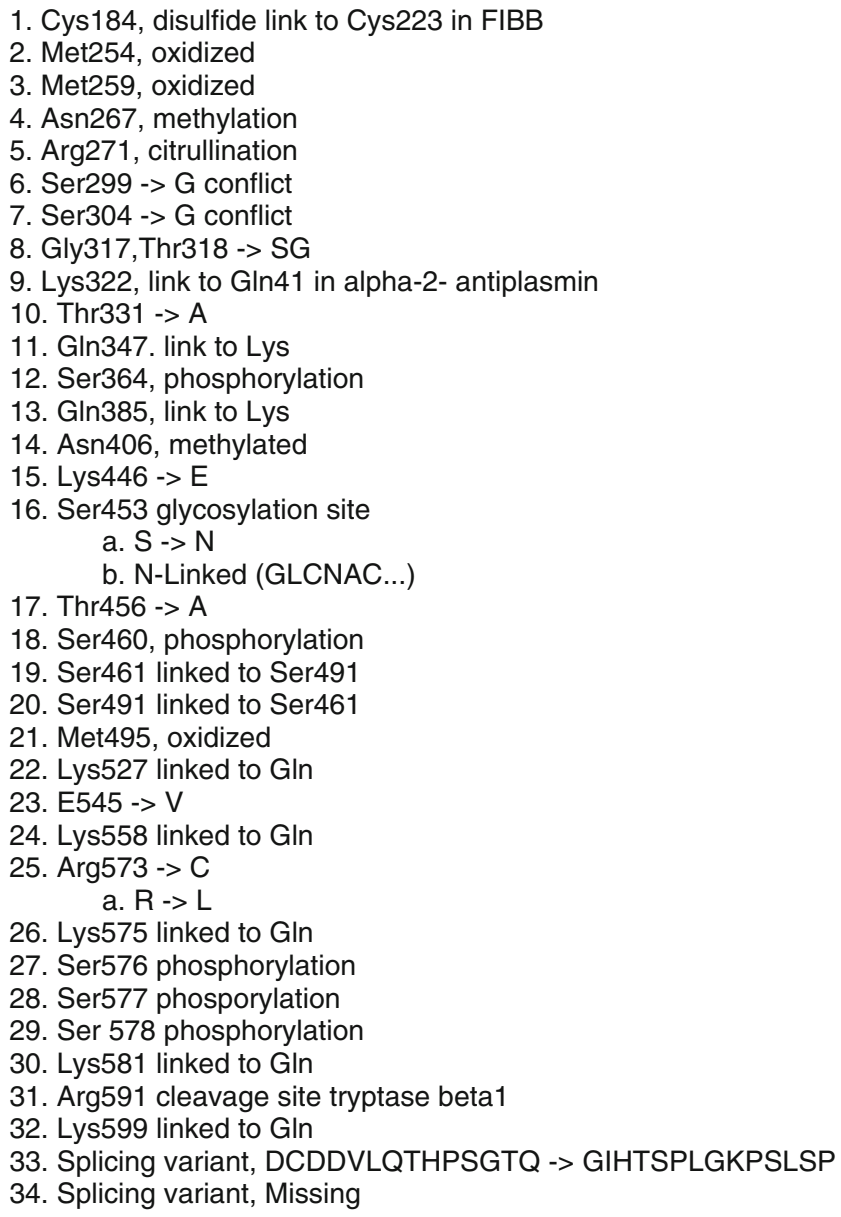

consistent with observed mass values of this FIBA fragment in RA SF and the failure of trypsin to cleave at that site.

As a final proof that the citrullination site was correctly assigned, the peptide corresponding to residues 259-287 was synthesized with and without the citrulline in position 271 (designated $271 \mathrm{X}$ and $271 \mathrm{R}$, respectively). Both the charge state distribution in the electrospray spectrum and the fragment masses in the MS/MS spectrum of the $3+$ charge state of $271 \mathrm{X}$ matched spectral data obtained with the sample isolated from RA SF.

To establish that the citrullinated FIBA 259-287 peptide was recognized specifically by RA patient sera, the two FIBA 259-287 synthetic peptides were tested in an ELISA. An additional citrullinated synthetic peptide, corresponding to profilaggrin 619-631 (FIL) with a citrulline substitution at Arg 625, was included as a control. The immobilized peptides were incubated with sera from RA, SLE, or healthy controls, followed by detection of bound antibodies by HRP-conjugated anti-human $\operatorname{IgG}$, IgA, and $\operatorname{IgM}$ antibodies and a colorimetric assay. Graphs depicting the
ELISA optical density readings are shown in Fig. 6. Of 18 healthy control sera tested, two reacted to the $271 \mathrm{R}$ peptide, two reacted to the $271 \mathrm{X}$ peptide, and one reacted to the FIL peptide. Of the 12 RA sera tested, four reacted to the $271 \mathrm{R}$ peptide, 10 reacted to the $271 \mathrm{X}$ peptide, and three reacted to the FIL peptide. Of the 10 SLE sera tested, one patient reacted to all three peptides. The number of sera that reacted exclusively to the $271 \mathrm{X}$ peptide, and not with the 271R nor FIL peptides, was 5/12 RA sera, 0/18 healthy sera, and 0/10 SLE sera. These results provide evidence that antibodies in a subset of RA sera bind specifically to the citrulline residue at position 271 of the FIBA alphaC domain found in RA SF.

\section{Discussion}

Biomarker analysis consists of three phases: discovery, verification, and validation [38, 39]. Discovery is performed by a thorough analysis of a few samples with hopes of identifying many candidate biomarkers to be used for 


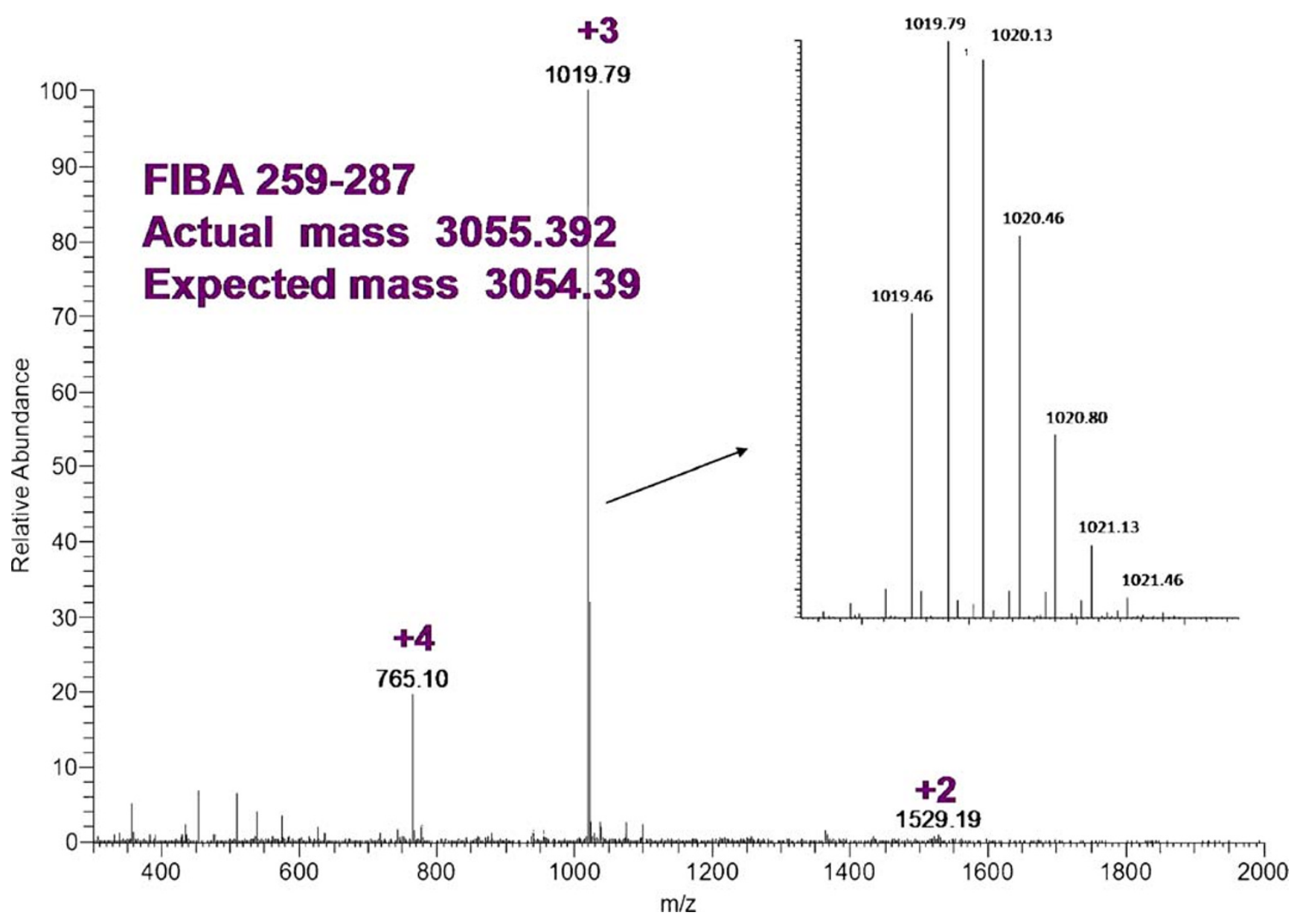

Fig. 5 Electrospray mass spectra of the citrullinated FIBA 259-287 peptide. The upper spectrum shows the observed mass value for each of the indicated charge states. The inset shows the isotope distribution for the $3+$ charge state

Table 2 The sequence for the citrullinated peptide along with other fragments for which high-resolution MS and MS/MS spectra were obtained

\begin{tabular}{|c|c|c|c|c|l|l|l|}
\hline $\mathbf{z}$ & $\mathbf{M}$ (obs) & $\mathbf{M}$ (pep) & $\mathbf{d M}$ & $\mathbf{T}$ & Peptide & aa\# & Mods \\
\hline 2 & 1500.742 & 1500.74 & 0 & $\mathrm{~F}$ & MELERPGGNEITR & $259-271$ & \\
\hline 3 & 1516.743 & 1500.74 & 16 & $\mathrm{~F}$ & MELERPGGNEITR & $259-271$ & oxidation \\
\hline 2 & 1344.644 & 1344.63 & 0 & $\mathrm{P}$ & MELERPGGNEIT & $259-270$ & \\
\hline 2 & 1360.634 & 1344.63 & 16 & $\mathrm{P}$ & MELERPGGNEIT & $259-270$ & oxidation \\
\hline 4 & 3053.396 & 3054.39 & 1 & $\mathrm{~F}$ & MELERPGGNEITRGGSTSYGTGSETESPR & $259-287$ & citrullination \\
\hline 3 & 3055.38 & 3054.39 & 1 & $\mathrm{~F}$ & MELERPGGNEITRGGSTSYGTGSETESPR & $259-287$ & citrullination \\
\hline 2 & 1240.66 & 1240.66 & 0 & $\mathrm{P}$ & LERPGGNEITR & $261-271$ & \\
\hline 2 & 1240.66 & 1240.66 & 0 & $\mathrm{P}$ & LERPGGNEITR & $261-271$ & \\
\hline 3 & 2795.29 & 2794.31 & 1 & $\mathrm{P}$ & LERPGGNEITRGGSTSYGTGSETESPR & $261-287$ & citrullination \\
\hline 2 & 1127.576 & 1127.57 & 0 & $\mathrm{P}$ & ERPGGNEITR & $262-271$ & \\
\hline 2 & 2398.044 & 2396.08 & 2 & $\mathrm{~F}$ & PGGNEITRGGSTSYGTGSETESPR & $264-287$ & deamindation \& citrullination \\
\hline 3 & 2423.088 & 2299.03 & 124 & $\mathrm{P}$ & GGNEITRGGSTSYGTGSETESPR & $265-287$ & acetylation \\
\hline 2 & 2071.926 & 2070.95 & 1 & $\mathrm{P}$ & EITRGGSTSYGTGSETESPR & $268-287$ & citrullination \\
\hline 3 & 2071.935 & 2070.95 & 1 & $\mathrm{P}$ & EITRGGSTSYGTGSETESPR & $268-287$ & citrullination \\
\hline 2 & 1571.67 & 1571.67 & 0 & $\mathrm{~F}$ & GGSTSYGTGSETESPR & $272-287$ & \\
\hline
\end{tabular}

The $\mathrm{dM}$ indicates the nominal mass difference between the expected and observed mass for the unmodified precursor ion. Possible sites of posttranslational modification are indicated in red. Only citrullination of the Arg residue is consistent with the observed mass values and the failure of trypsin to cleave at that site 
a

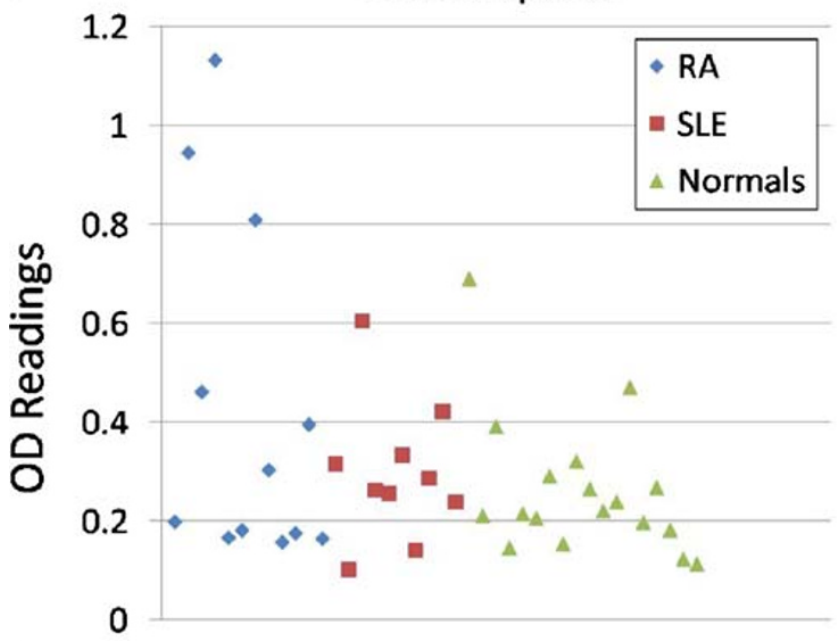

b

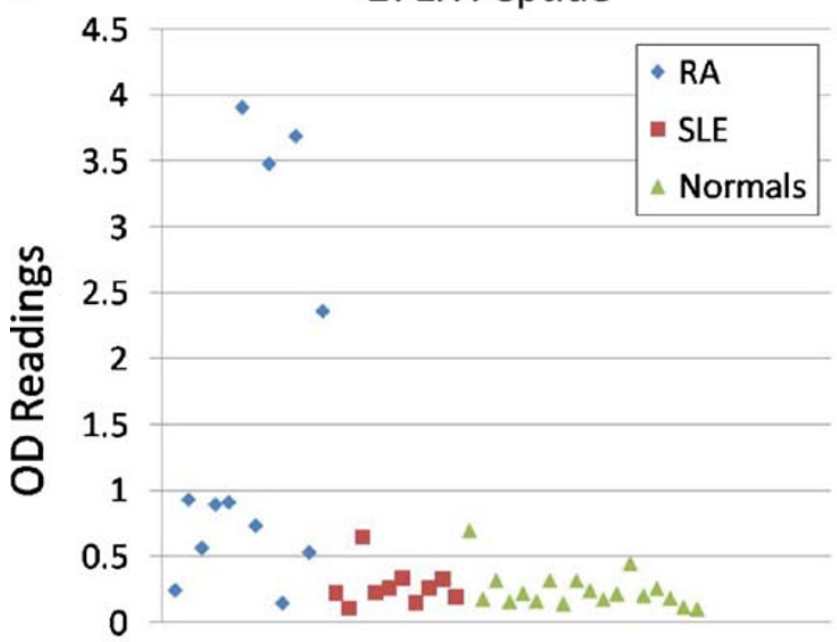

C

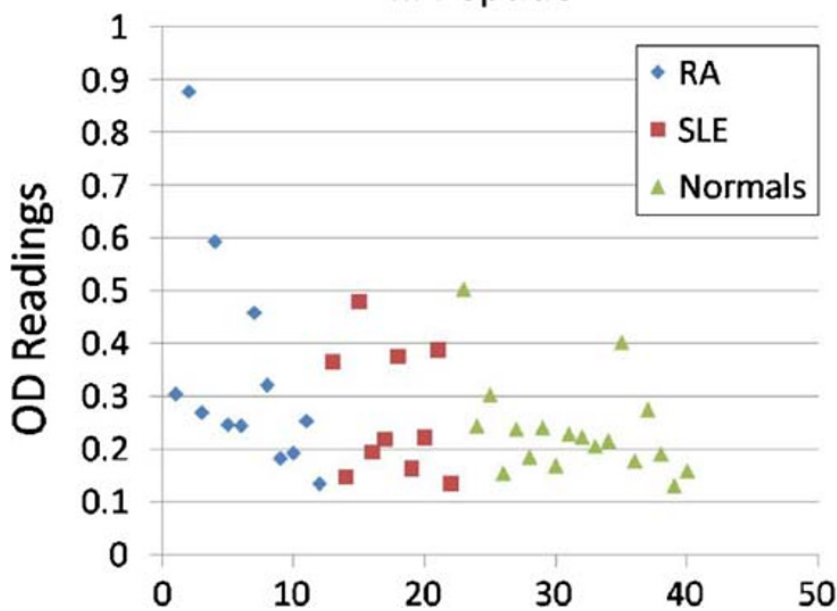

further study. Verification is performed by determining the presence of select candidate biomarker in a large number of clinical samples to estimate sensitivity and specificity. Sensitivity is defined as the percentage of patients with
Fig. 6 RA sera contain antibodies that specifically bind FIBA peptides bearing a citrulline substitution at Arg 271. In an ELISA, RA, SLE or control (C) sera were incubated with plate-immobilized peptides corresponding to FIBA 259-287 with (271X) or without (271R) a citrulline at position 271, or to filaggrin 619-631 (fil) with a citrulline at position 625 . Sera that reacted with 271R include: two out of 18 normals, four out of 12 RA, one out of 10 SLE. Sera that reacted with 271X include: two out of 18 normals, 10 out of $12 \mathrm{RA}$, one out of 10 SLE. Sera that reacted with fil include: one out of 18 normals, three out of 12 RA, one out of 10 SLE

the specified disease that test positive for the biomarker. Specificity is defined as the percentage of people that do not have the specified disease that test negative for the biomarker. Candidate biomarkers that have a high sensitivity and high specificity are further analyzed in the validation phase. Validation is performed by analyzing the presence of the candidates in a patient pool that is expected to be present in a clinical setting where patients with the specific disease will be diagnosed. The purpose of validation is to test whether the candidate can be used for diagnosis. After validation, the biomarkers may be used to develop a diagnostic test. This study represents the discovery phase of biomarker analysis.

We have used a proteomic method to fractionate RA SF proteins, determine their reactivity to autoantibodies in RA sera, and identify immunogenic antibody epitopes. This method involves depletion of abundant serum proteins, twodimensional liquid chromatography, protein macroarrays probed with RA and control sera to identify fractions containing potential autoantigens, and mass spectrometric analyses of those fractions via high-resolution LC-MS/MS. We identified a portion of a SF protein, the fibrin alphaC domain fragment, whose immunogenicity depended upon an Arg to citrulline post-translational modification that had occurred in vivo. We have shown that RA autoantibodies specifically target an epitope containing citrulline at position 271 of FIBA and that this post-translational modification of Arg 271 is present in RA SF. This approach is a feasible strategy that can be used to identify or confirm other autoantigens in RA SF as well as self-proteins that are targets of autoreactive $\mathrm{B}$ cell responses in other autoimmune diseases.

Citrullinated fibrinogen is a known autoantigen in RA $[17,40]$. Experiments using purified fibrinogen and PAD enzymes in vitro identified 22 possible citrullination sites in FIBA [41, 42]; the Arg 271 identified in our study was citrullinated by both PADI4 and PADI2 enzymes in vitro. Several groups have reported reactivity of RA sera to synthetic FIBA peptides [19, 43, 44] and fibrinogen present in synovial exosomes [18]. Another report showed that mAbs specific for the same citrullinated FIBA peptide identified in our study detected the peptide epitope's presence in RA SF but not RA plasma [19]. The results from our study confirm the presence and reactivity 
of citrullinated fibrin/fibrinogen in SF. If our study had been performed using RA plasma as the source of autoantigens, we would not have been successful at identifying autoantigens. Identifying the best biomarker source is crucial in biomarker discovery.

It is possible that our study failed to detect additional immunogenic citrulline sites on FIBA as well as other molecules. The failure of trypsin to cleave at citrulline residues often results in large peptides that are difficult to characterize by mass spectrometry. Other autoantigens could be present at very low concentrations, lost due to non-specific binding during chromatography, did not bind to the nitrocellulose, did not bind in the right conformation to the nitrocellulose, are heavily modified or glycosylated, were undetected by the antibodies in the macroarrays, and/ or were not completely digested by trypsin. For these and many more reasons, the proteins could have escaped detection by the macroarray or mass spectrometry experiments. This study was designed to identify proteins so if the autoantigens are carbohydrate, lipid, or another type of molecule then they would have also been undetected. The chromatographs are more sensitive at detecting proteins than the mass spectrometry. However, since small chromatography peaks yielded no protein identifications, the nature of the molecule that produced the peak is unknown.

The role for the fibrin alphaC domain fragment in RA pathogenesis may be complex. Soluble citrullinated fibrinogen and fibrin degradation products have been found in RA SF but not RA plasma, suggesting that there are high levels of active PAD enzymes in RA SF [19, 45]. Fibrin deposits in the joints of RA patients are widely observed and have been hypothesized to be the cause of pannus formation [46]. The fibrin deposits in the joints allow the fibrin molecules to remain in an inflammatory environment for a prolonged period of time, which could facilitate posttranslational citrulline modification [5]. The alphaC domain fragments are the first to be released during fibrinolysis so they are constantly being generated [47]. If stably present, such degraded alphaC fragments could readily be taken up by antigen presenting cells and chronically displayed to the immune system. Hence, a degradation product of an aberrantly modified self-protein may be an RA autoantigen.

This discovery of post-translationally modified immunogenic epitopes present on self-proteins in vivo may contribute to improved diagnostic tests for RA. With the data obtained from a limited number of patients, the citrullinated 271X peptide seems to have similar sensitivity $(83.3 \%)$ as the commercially available CCP2 test. Since antibodies to CCP have been shown to be present prior to disease-onset development $[11,12]$, there is a possibility that the citrullinated $271 \mathrm{X}$ peptide can also predict disease. Studies determining when autoantibodies to the citrullinated 271X are produced need to be conducted. Whether a test using the native peptide offers any improvement over the commercially available tests remains to be shown. Irreversible joint damage can occur early in the disease process [48], so early diagnosis and aggressive treatment is vital to the preservation of joint function. Autoantibodies specific for citrullinated epitopes are predominant in early RA patients with high-grade joint inflammation and clinical manifestations predicting development of severe erosive disease $[43,49]$. One promising diagnostic tool to define clinically distinct subsets of RA patients is antigen microarray profiling of autoantibodies, an assay in which known autoantigens are arrayed on slides, which are probed with patient sera [43]. The method described here, which can be used to identify epitopes on proteins that are modified in inflamed synovial tissue in situ, will lead to additional information about autoantigens that will help to increase the power of such diagnostic autoantigen arrays.

In summary, proteomic analysis defined an immunogenic citrulline-containing epitope, within the fibrin alphaC domain fragment, as an autoantigen present in RA SF. This study provides further validation that citrullinated fibrinogen is an autoantigen in RA. The strategy used in this project should be useful for identification of novel autoantigens in RA and other autoimmune diseases.

Acknowledgements The authors would like to thank Drs. Andrew J. Alpert, Alex Kurosky, Paul Plotz, and Henry M. Fales for reviewing the manuscript, Dr. Robert Nathan for discussions on array analysis, Dr. Leonid Medved for providing the structure of the alphaC domain, and the patients and volunteers who donated the serum and SF samples.

\section{References}

1. Feldmann M, Brennan FM, Maini RN. Rheumatoid arthritis. Cell. 1996;85:307-10.

2. Firestein GS. Evolving concepts of rheumatoid arthritis. Nature. 2003;423(6937):356-61.

3. Corrigall VM, Panayi GS. Autoantigens and immune pathways in rheumatoid arthritis. Crit Rev Immunol. 2002;22 (4):281-93.

4. Boekel MAv, et al. Autoantibody systems in rheumatoid arthritis: specificity, sensitivity and diagnostic value. Arthritis Rheum. 2002;4:87-93.

5. Gaalen Fv, et al. The devil in the details: the emerging role of anticitrulline autoimmunity in rheumatoid arthritis. J Immunol. 2005; 175:5575-80.

6. Firestein G. Immunologic mechanisms in the pathogenesis of rheumatoid arthritis. J Clin Rheumatol. 2005;11:S39-44.

7. Utz PJ, Gensler TJ, Anderson P. Death, autoantigen modifications, and tolerance. Arthritis Res Ther. 2000;2:101-14.

8. Vossenaar ER, Venrooij WJv. Citrullinated proteins: sparks that may ignite the fire in rheumatoid arthritis. Arthritis Res Ther. 2004;6:107-11.

9. Plotz P. The autoantibody repertoire: searching for order. Nat Rev Immunol. 2003;3:73-8. 
10. Schellekens GA, et al. Citrulline is an essential constituent of antigenic determinants recognized by rheumatoid arthritis-specific autoantibodies. J Clin Invest. 1998;101:273-81.

11. Rantapää-Dahlqvist S, et al. Antibodies against cyclic citrullinated peptide and iga rheumatoid factor predict the development of rheumatoid arthritis. Arthritis Rheum. 2003;48(10):2741-9.

12. Nielen MMJ, et al. Specific autoantibodies precede the symptoms of rheumatoid arthritis. Arthritis Rheum. 2004;50(2):380-6.

13. Lundberg K, et al. Citrullinated proteins have increased immunogenicity and arthritogenicity and their presence in arthritic joints correlates with disease severity. Arthritis Res Ther. 2005;7:R458-67.

14. Mil AHvdH-v, et al. Antibodies to citrullinated proteins and differences in clinical progression of rheumatoid arthritis. Arthritis Res Ther. 2005;7:R949-58.

15. Suzuki A, Yamada R, Chang X, Tokuhiro S, et al. Functional haplotypes of PADI4, encoding citrullinating enzyme peptidylarginine deiminase 4, are associated with rheumatoid arthritis. Nat Genet. 2003;34:395-402.

16. Tarcsa E, et al. Protein unfolding by peptidylarginine deiminase. $\mathrm{J}$ Biol Chem. 1996;271(48):30709-16.

17. Masson-Bessière $\mathrm{C}$, et al. The major synovial targets of the rheumatoid arthritis-specific antifilaggrin autoantibodies are deiminated forms of the a- and b-chains of fibrin. J Immunol. 2001;166:4177-84.

18. Skriner K, et al. Association of citrullinated proteins with synovial exosomes. Arthritis Rheum. 2006;54(12):3809-14.

19. Takizawa Y, et al. Citrullinated fibrinogen detected as a soluble citrullinated autoantigen in rheumatoid arthritis synovial fluids. Ann Rheum Dis. 2006;65:1013-20.

20. Girbal-Neuhauser E, et al. The epitopes targeted by the rheumatoid arthritis-associated antifilaggrin autoantibodies are posttranslationally generated on various sites of (Pro) filaggrin by deimination of arginine residues. J Immunol. 1999;162:585-94.

21. Sebbag M, et al. The antiperinuclear factor and the so-called antikeratin antibodies are the same rheumatoid arthritis-specific autoantobodies. J Clin Invest. 1995;95:2672-9.

22. Vossenaar ER, et al. Rheumatoid arthritis specific anti-Sa antibodies target citrullinated vimentin. Arthritis Res Ther. 2004;6:R142-50.

23. Bang $\mathrm{H}$, et al. Mutation and citrullination modifies vimentin to a novel autoantigen for rheumatoid arthritis. Arthritis Rheum. 2007;56(8):2503-11.

24. Tilleman K, et al. Synovial detection and autoantibody reactivity of processed citrullinated isoforms of vimentin in inflammatory arthritides. Rheumatology (Oxford). 2008;47(5):597-604.

25. Suzuki A, et al. Anti-citrullinated collagen type I antibody is a target of autoimmunity in rheumatoid arthritis. Biochem Biophys Res Commun. 2005;333:418-26.

26. Koivula MK, et al. Sensitivity and specificity of autoantibodies binding to citrullinated carboxyterminal telopeptides of types I and II collagens in an early arthritis series. Rheumatology (Oxford). 2008;47(5):656-9.

27. Burkhardt $\mathrm{H}$, et al. Humoral immune response to citrullinated collagen type II determinants in early rheumatoid arthritis. Eur J Immunol. 2005;35:1643-52.

28. Yoshida M, et al. Autoimmunity to citrullinated type II collagen in rheumatoid arthritis. Mod Rheumatol. 2006;16(5):276-81.

29. Chang $X$, et al. Citrullination of fibronectin in rheumatoid arthritis synovial tissue. Rheumatology. 2005;44(11):1374-1382.
30. Kinloch A, et al. Identification of citrullinated $\alpha$-enolase as a candidate autoantigen in rheumatoid arthritis. Arthritis Res Ther. 2005; 7:R1421-9.

31. Hueber W, Robinson WH. Proteomic biomarkers for autoimmune disease. Proteomics. 2006;6:4100-4115.

32. Yan F, et al. Protein microarrays using liquid phase fractionation of cell lysates. Proteomics. 2003;3:1228-35.

33. Arnett FC, et al. The American Rheumatism Association 1987 revised criteria for the classification of rheumatoid arthritis. Arthritis Rheum. 1988;31(3):315-24.

34. Hochberg MC, et al. The American College of Rheumatology 1991 revised criteria for the classification of global functional status in rheumatoid arthritis. Arthritis Rheum. 1992;35(5):498 502.

35. Smith DD, et al. Median filter algorithm for estimating the threshold of detection on custom protein arrays. Biotechniques. 2006;41:74-8.

36. Yates JRd, Eat J, McCormack AL, Schieltz D. Method to correlate tandem mass spectra of modified peptides to amino acid sequences in the protein database. Anal Chem. 1995;67:1426-36.

37. Chen EI, et al. Large scale protein profiling by combination of protein fractionation and multidimensional protein identification technology (MudPIT). Mol Cell Proteomics. 2006;5:53-6.

38. Rifai N, Gillette MA, Carr SA. Protein biomarker discovery and validation: the long and uncertain path to clinical utility. Nat Biotechnol. 2006;24(8):971-83.

39. Zolg W. The proteomic search for diagnostic biomarkers: lost in translation? Mol Cell Proteomics. 2006;5(10):1720-6.

40. Chang X, Yamada R, Suzuki A, Sawada T, Yoshino S, Tokuhiro $\mathrm{S}$, et al. Localization of peptidylarginine deiminase 4 (PADI4) and citrullinated protein in synovial tissue of rheumatoid arthritis. Rheumatology (Oxford). 2005;44(1):40-50.

41. Nakayama-Hamada $M$, et al. Comparison of enzymatic properties between hPADI2 and hPADI4. Biochem Biophys Res Commun. 2005;327:192-200.

42. Kubota K, Yoneyama-Takazawa T, Ichikawa K. Determination of sites citrullinated by peptidylarginine deiminase using ${ }^{18} \mathrm{O}$ stable isotope labeling and mass spectrometry. Rapid Commun Mass Spectrom. 2005;19:683-8.

43. Hueber W, et al. Antigen microarray profiling of autoantibodies in rheumatoid arthritis. Arthritis Rheum. 2005;52(9):2645-55.

44. Sebbag M, et al. Epitopes of human fibrin recognized by the rheumatoid arthritis-specific autoantibodies to citrullinated proteins. Eur J Immunol. 2006;36:2250-63.

45. Sinz A, et al. Mass spectrometric proteome analyses of synovial fluids and plasmas from patients suffering from rheumatoid arthritis and comparison to reactive arthritis or osteoarthritis. Electrophoresis. 2002;23:3445-56.

46. Sánchez-Pernaute O, et al. A fibrin based model for rheumatoid synovitis. Ann Rheum Dis. 2003;62:1135-8.

47. Henschen-Edman AH. Fibrinogen non-inherited heterogeneity and its relationship to function in health and disease. Annals NY Acad Sci. 2001;936:580-93.

48. Scott D. Radiological progression in established rheumatoid arthritis. J Rheumatol Suppl. 2004;69:55-65.

49. Gaalen FAv, et al. Autoantibodies to cyclic citrullinated peptides predict progression to rheumatoid arthritis in patients with undifferentiated arthritis. Arthritis Rheum. 2004;50(3):709-15. 